NBER WORKING PAPER SERIES

\title{
EFFECTS OF OPIOID-RELATED POLICIES ON OPIOID UTILIZATION, NATURE OF MEDICAL CARE, AND DURATION OF DISABILITY
}

\author{
David Neumark \\ Bogdan Savych \\ Working Paper 29371 \\ http://www.nber.org/papers/w29371 \\ NATIONAL BUREAU OF ECONOMIC RESEARCH \\ 1050 Massachusetts Avenue \\ Cambridge, MA 02138 \\ October 2021, Revised August 2022
}

We would like to thank Philip Borba, Marcus Dillender, John Ruser, Vennela Thumula, Dongchun Wang, and anonymous reviewers for valuable input, and Eric Harrison for assistance with the data. Any errors or omissions remaining in this study are the responsibility of the authors. The views expressed herein are those of the authors and do not necessarily reflect the views of the National Bureau of Economic Research.

NBER working papers are circulated for discussion and comment purposes. They have not been peerreviewed or been subject to the review by the NBER Board of Directors that accompanies official NBER publications.

(C) 2021 by David Neumark and Bogdan Savych. All rights reserved. Short sections of text, not to exceed two paragraphs, may be quoted without explicit permission provided that full credit, including @ notice, is given to the source. 
Effects of Opioid-Related Policies on Opioid Utilization, Nature of Medical Care, and Duration of Disability

David Neumark and Bogdan Savych

NBER Working Paper No. 29371

October 2021, Revised August 2022

JEL No. I13,J28

\begin{abstract}
We examine the effects of must-access prescription drug monitoring programs (PDMPs) and recent regulations limiting the duration of initial opioid prescriptions on care received by patients with work-related injuries, focusing on opioid utilization and medical care related to pain management. We find that must-access PDMPs contributed to declines in opioid utilization, while regulations limiting duration of initial opioid prescriptions had little effect on whether workers receive opioids, but reduced opioid use among those with prescriptions. We find some evidence that must-access PDMPs affected utilization of other medical care-most interestingly, in light of high opioid use, towards non-opioid pain medication and interventional pain management services for neurologic spine pain. We find that must-access PDMPs and limits on initial prescriptions had little impact on the duration of temporary disability benefits captured at 12 months of maturity.
\end{abstract}

David Neumark

Department of Economics

University of California, Irvine

3151 Social Science Plaza

Irvine, CA 92697

and NBER

dneumark@uci.edu

Bogdan Savych

Workers Compensation Research Institute

955 Massachusetts Ave.

Cambridge, MA

02139

bsavych@gmail.com 


\section{Introduction}

The last decade saw extensive policy focus on opioid abuse and prescribing. Policies adopted included implementation of electronic prescription drug monitoring programs (PDMPs), which first encouraged and later mandated their use before a prescription could be issued, and, more recently, limits on durations of initial opioid prescriptions. We examine the effects of these state-level policies on utilization of opioids, the nature of care (i.e., substitution towards other kinds of care), and the duration of temporary disability benefits among workers recovering from work-related injuries, exploring their effects in the workers' compensation system. There is other research studying the effects of opioid-related policies on opioid prescribing behavior and use, but to the best of our knowledge, we are the first to provide comprehensive evidence on the effects of these policies, across states, in the workers’ compensation setting. ${ }^{1}$ However, our work looking beyond prescribing behavior, and in particular at substitution towards other types of medical care induced by policies intended to restrict opioids, in novel.

Opioid medications provided in the workers' compensation system are a significant contributor to opioid prescribing overall—including longer-term prescribing—among working adults. Opioid use is common among workers injured at work. Recent studies show that more than half of injured workers who are absent from work more than seven days, who do not receive surgery but receive pain medications, received an opioid prescription. Many of these workers received opioids on a longer-term basis (Thumula et al., 2017). Work-related injuries represent a substantial share of injuries that occur to working adults. Nearly half of all trauma injuries to working adults in 2008-2010 were deemed to be work-related and were covered by workers' compensation insurance, and one in five injuries for soft-tissue conditions were deemed work-related (Victor et al., 2015).

The adoption of the policies we study in this paper coincided with a decline in opioid utilization

\footnotetext{
${ }^{1}$ Thumula (2017) examined the impact of Kentucky's House Bill 1 that introduced regulations for pain clinics and established standards for dispensing and prescribing of opioids, including mandating use of the state PDMP, on opioid prescriptions for workers' compensation injuries. Outside of workers' compensation, there is one study of policies limiting initial opioid prescriptions in a group health setting (Sacks et al., 2019), and there is research on PDMPs and other earlier opioid-related policies in the group health and Medicare/Medicaid settings (see reviews by Maclean et al., 2020, and Ben-Shalom et al., 2020). However, all of this work focuses on opioid utilization. Our work differs in looking at opioid-related policies across many states, in the workers' compensation setting.
} 
in the workers' compensation system, although opioid use remains common among workers injured at work (Thumula et al., 2019; Hayes and Swedlow, 2019; Texas Department of Insurance, 2019). Figure 1 shows that opioid use has declined substantially since 2010. The top panel shows a decline in the morphine milligram equivalent amount (MME) of opioids prescribed per claim, and the bottom panel shows a decline in the percentage of claims with opioids. Both graphs show the data for all claims and for the subset of claims with more than 7 days of lost work time (which entail more serious injuries and hence higher values for both measures). Between 2010 and 2018, the average MME amount of opioids prescribed per claim at 12 months of maturity decreased 74 percent. This reflects decreases in the percentage of workers with injuries who were prescribed opioids as well as the amount of opioids prescribed per claim with opioids. As the bottom graph shows, 10 years ago nearly half of claims with more than 7 days of lost work time had an opioid prescription, as did nearly 20 percent of all claims. These percentages dropped to about 30 percent and 10 percent, respectively, by $2018 .{ }^{2}$ Nevertheless, many workers continue to receive opioids on a prolonged basis. Across 22 states, in 2016, among workers who received opioid prescriptions, the percentage that received at least 60 days of opioids supply over any 90-day period ranged from 6 percent to 33 percent (Thumula et al., 2019).

These policy developments raise several important questions and concerns. First, the most direct question is how opioid-related policies contributed to changes in opioid utilization. We examine this question by looking at a number of measures of opioid prescribing. ${ }^{3}$ These measures include whether workers had any opioid prescriptions; the number of prescriptions; the MME amount of opioids prescribed; and whether workers experienced potentially problematic prescriptions (i.e., longer-term prescribing of opioids within 12 months of injury, ${ }^{4}$ more than 90 days of opioids prescribed, or more than

\footnotetext{
${ }^{2}$ Note that the years indicated in the figure reflect injury year, and are evaluated at 12 months of experience, which actually extends into the next calendar year.

${ }^{3}$ We examine opioid prescriptions filled by workers with injuries that were paid for by workers' compensation payors. We do not observe whether workers actually take opioids, and we do not observe prescriptions filled outside the workers' compensation system.

${ }^{4}$ We measure longer-term prescribing as opioid prescriptions within the first three months after an injury and three or more filled opioid prescriptions between the 7th and 12th months after an injury.
} 
$120 \mathrm{mg}$ morphine equivalent daily dose of opioids prescribed). ${ }^{5}$

Second, the potential for reductions in opioid prescribing raises the question of how workers with injuries managed their pain. Opioid prescribing and pain treatment guidelines recommend adoption of alternatives to opioids when treating acute and chronic pain (Centers for Disease Control and Prevention [CDC], 2016; Hegmann et al., 2014; American College of Occupational and Environmental Medicine, 2017). These alternatives include non-opioid pain medications (e.g., nonsteroidal anti-inflammatory drugs and non-opioid analgesics), as well as non-pharmacologic treatment (e.g., active and passive physical medicine, chiropractic care, acupuncture, and cognitive behavioral services). However, the adoption of these alternatives in response to policies intended to reduce opioid prescribing has not been examined. One important exception that looks at substitution from a different angle is Mallatt (2022), who studies effects of opioids-reduction policies (and the reformulation of OxyContin) on substitution towards heroin, based on crime data on heroin possession and dealing. ${ }^{6}$

In our analyses, we study whether workers received other care that may be a substitute for opioid therapies. Following recommendations in treatment guidelines, we examine whether workers shift to nonopioid pain medications, to interventional pain management treatment, or to use of active physical medicine services. We study substitution across types of care for a work-related injury or illness, and we focus on care for broadly-defined groups of injuries (e.g., low-back cases, fractures, lacerations and contusions, or inflammations). ${ }^{7}$ The focus on these groups of injuries is beneficial because opioid prescribing rates differ widely across injury groups, with the highest opioid utilization among neurologic spine pain cases. Furthermore, the responsiveness to policies also likely differs by injury group, since treatment guideline recommendations regarding prescribing of opioids, and the appropriateness of alternative treatments, differ depending on the type of injury. For example, evidence-based treatment

\footnotetext{
${ }^{5}$ These measures were used in Thumula et al. (2019) and Sacks et al. (2019). Data limitations prevent us from examining other measures that may indicate an increased risk of abuse, such as the number of prescribers who prescribed opioids or the number of pharmacies that dispensed prescriptions.

${ }^{6}$ This paper builds on the analysis in Mallatt (2018).

${ }^{7}$ In workers' compensation, substitution among services is not expected to be influenced by prices paid by consumers (workers), as there are no co-pays or deductibles. However, the costs of the services and reimbursements for those costs can affect provider behavior (Savych and Fomenko, 2019; Yee et al., 2015).
} 
guidelines recommend against long-term use of opioids for low-back cases (e.g., Bigos et al., 1994).

Finally, we examine whether opioid-related policies affected the duration of temporary disability benefits. Workers receive temporary disability benefits when they cannot work while recovering from injuries, and hence the duration of temporary disability benefits is strongly correlated with the length of time until return to work (although not identical). An increase in the duration of temporary disability benefits as a result of opioid-related policies may indicate that limits on access to opioid therapies may have delayed workers’ recovery after an injury, or vice versa. ${ }^{8}$

We find that must-access PDMPs contributed to a decline in the amount of opioids prescribed and the number of opioid prescriptions, while there was little change in whether workers received opioids. We also find that must-access PDMPs contributed to a decrease in the likelihood that workers receive opioids on a longer-term basis, among those with opioids. We find that limits on initial opioid prescriptions reduced opioid use among claims with opioids. We find some evidence that workers increased the use of other types of care when policies restricted access to opioid prescriptions. Most interesting, in our view, is the evidence for neurologic spine pain cases, where we find evidence consistent with partial substitution towards non-opioid pain medication prescriptions and interventional pain management services. This is potentially significant because, among the injury groups we consider, neurologic spine pain cases have the highest incidence of opioid prescriptions and the highest MME. Finally, we do not find evidence that either must-access PDMPs or limits on initial prescribing resulted in changes in the duration of temporary disability benefits, although the estimates are not very precise and hence do not rule out reductions that could be meaningful.

\section{Opioid-Related Policies and Prior Evidence}

Increased attention to opioid prescribing by policymakers, payors, and providers in response to

\footnotetext{
${ }^{8}$ Physicians play an important role in deciding the end of the period of temporary disability, as they decide whether workers have fully recovered, whether they can be released back to work, and whether they still have some remaining disability. Specific processes for ending temporary disability benefits vary across states, especially if there are disputes about workers' ability to go back to work. We are not aware of any specific guidance about how opioid prescribing should be related to doctors' decisions regarding disability. Opioid prescriptions for acute pain may indicate that underlying disability has not been resolved.
} 
the opioid crisis was likely an important contributor to the declines in opioid prescribing documented in Figure 1. A flurry of state-level policies and regulations—-some part of the workers' compensation system, and some more general—were implemented to help prevent opioid abuse.

The policies that targeted various dimensions of opioid prescribing include (but are not limited to): must-access PDMPs; limits on initial opioid prescriptions; general as well as pain treatment guidelines; mandatory prescriber education and physician licensing; pain clinic regulations; and state workers’ compensation regulations about dispensing and utilization of opioids (i.e., drug formularies). Our analysis focuses on two policies: must-access PDMPs, which were shown to be effective in reducing opioid use in the general health setting (although there are no studies of the effects of these policies in workers' compensation); and limits on initial opioid prescriptions, which have not been examined much in the prior literature. There were also concurrent federal policy interventions, including CDC guidelines (CDC, 2016), changes in the Controlled Substance Act (CSA), and Risk Evaluation and Mitigation Strategies (REMS) programs. In this study, we focus on state policies, in part because it is more difficult to identify the effects of federal policy changes since they were enacted everywhere and hence can be confounded with other sources of changes in opioid prescribing. Our analysis estimates the effects of these state policies above and beyond any effects of the federal policies.

\section{A. PRESCRIPTION DRUG MONITORING PROGRAMS}

PDMPs may be used by states to identify excessive prescribing of opioids and potential opioid abuse. These programs create a centralized listing of prescriptions that gives health care providers information on patients' prescription histories. PDMPs are intended to limit access to prescription drugs when individuals are likely to misuse them—as evidenced by multiple prescriptions from multiple prescribers at the same time-ideally leading to a reduction in prescription opioids among those with higher use. At the same time, in principle PDMPs should not limit access to opioid prescriptions when the medications are used appropriately. ${ }^{9}$ PDMP programs differ across states in a number of dimensions,

\footnotetext{
${ }^{9}$ Some studies do, however, suggest that PDMPs may also contribute to reduction in opioid use due to the hassle costs they pose (Alpert et al., 2020; Sacks et al., 2019).
} 
including: what information is collected in the database; who should report prescriptions and how often; whether providers are required to check the PDMP; whether the PDMP is implemented electronically; and who can and should access the PDMP and for what purpose (Thumula et al., 2019).

Evidence from the general health setting indicates that PDMPs are effective in reducing opioid prescribing when states require that medical providers access PDMPs before issuing a new prescription, while voluntary use PDMPs did not affect opioid use. ${ }^{10}$ This difference likely reflects increased utilization of PDMPs when states mandate their use (Pew Charitable Trusts, 2016), while, in contrast, voluntary PDMPs did not create strong incentives for providers to change their prescribing behavior. ${ }^{11}$ We thus focus our estimates on adoption of must-access PDMPs as one of the policies of interest in our analysis.

Table 1 provides information about timing of the policies we examine. The first column shows when states implemented must-access PDMPs. We use the dates of policy implementation drawn primarily from Davis et al. (2019) and Thumula et al. (2019), supplementing with our own investigation when the timing of policies was inconsistent with other sources of information on effective dates of policies. Prior studies show that choices about how to date the timing of policies contributed to differences in the estimated effects of the policies (Horwitz et al., 2018), such as dates of adoption versus implementation of access to PDMPs. (We study the effects of must-access PDMPs, but from here we often refer to these as simply "PDMPs.”) We use the dates the PDMPs became effective; there is less uncertainty about interpreting these dates than the dates policies were enacted.

\section{B. LIMITS ON INITIAL OPIOID PRESCRIPTIONS}

Policies introducing limits on initial opioid prescriptions respond to the concern that a larger number of days of opioids supplied in new prescriptions to treat acute pain may contribute to longer-term use of opioids (Shah et al., 2017). These policies limit the initial opioid prescriptions—-most commonly to 7 days of supply, although some states use limits of 3, 5, or 14 days of supply. A few states also limit the

\footnotetext{
${ }^{10}$ See, e.g., Bao et al. (2018), Buchmueller and Carey (2018), Grecu et al. (2019), Kaestner and Ziedan (2019), Mallatt (2018), Meinhofer (2018), Wen et al. (2019), and Ziedan and Kaestner (2020).

${ }^{11}$ The effects of must-access PDMPs may also or instead reflect the implementation of electronic access to programs amidst a wider sharing of electronic records in the medical field (Kaestner and Ziedan, 2019; Wang, 2021).
} 
morphine equivalent daily dose that can be prescribed per day (North Carolina, Nevada, Tennessee), or specify that prescriptions are limited to the lowest effective dose (Maryland). As Table 1 shows, Massachusetts was the first state to limit initial opioid prescriptions, in 2016. By end of March 2018, 14 of our analysis states (out of 33 total) introduced such limits, and a few more states introduced them subsequently. Thus, for most states we only capture the early experience after limits were implemented. ${ }^{12}$ Furthermore, in many states these policies were implemented after the CDC published voluntary guidelines for primary care practitioners that recommend the "lowest effective dose” when deciding on prescriptions for opioids. The CDC guidelines indicate that 3 days or fewer of opioids will often be sufficient for treating acute pain, and more than 7 days will rarely be needed (CDC, 2016). While these guidelines are voluntary, they may have affected prescribing behavior across all states, thus limiting the effectiveness of state limits on initial opioid prescriptions after the CDC guidelines were issued.

There is limited evidence about the effects of prescribing limits in the general health setting. Studies indicate a decrease in the percentage of opioid prescriptions above the days of supply limit after states implemented prescribing limits (Agarwal et al., 2019; Hincapie-Castillo et al., 2020). Sacks et al. (2019) compared trends in prescribing among states with and without the policies and found that limits on initial prescriptions appear to have reduced the length of initial opioid prescriptions for a population of patients with group health insurance, and increased the likelihood of short prescriptions. However, they also found an increase in the likelihood of opioid prescriptions dispensed to new users.

\section{OTHER OPIOID-RELATED POLICIES}

Other state policies related to opioid treatment also have been implemented. State and national treatment guidelines highlight preferred approaches to prescribing practices. ${ }^{13}$ Treatment guideline

\footnotetext{
${ }^{12}$ Policies that limit prescribing vary in other dimensions, including the types of prescriptions affected (whether the limits apply to all prescriptions, acute pain prescriptions, or only a subset of opioids). The policies also vary with respect to exceptions granted-including exceptions for chronic pain, cancer, substance use disorder treatment, or surgical pain, and exceptions based on a clinician's professional judgment (Thumula et al., 2019). We do not examine the differential effects of these variations in prescribing limits.

${ }^{13}$ The national guidelines include the CDC guidelines for prescribing opioids for chronic pain, the general treatment guidelines of the American Pain Society and the American Academy of Pain Management, the occupational medical treatment guidelines of the American College of Occupational and Environmental Medicine (ACOEM), and the Official Disability Guidelines (ODG) by MCG Health.
} 
approaches vary widely. Some state workers' compensation systems adopted general treatment guidelines that outline evidence about appropriate care, including use of opioids. Other states have adopted specific guidelines related to chronic pain and pain management. An important dimension of these policies is whether treatment guidelines are required to be followed and whether the state has mandatory utilization review to confirm that the care follows the guidelines. In general, guidelines discourage the use of opioids early after an injury, except for traumatic injuries or for patients with severe pain. When opioids are prescribed, the guidelines may recommend limits on the duration of prescriptions, ${ }^{14}$ limits on maximum daily dose, ${ }^{15}$ and checking PDMPs when prescribing opioids (Wang, 2017, Appendix B). Overall, there is limited evidence about the effects of adoption of medical treatment guidelines (Haegerich et al., 2014).

Some states have implemented drug formularies to directly target prescribing behavior in the workers' compensation system. These regulations often require additional approval steps for a subset of drugs (often including opioids along with many other medications). Evidence indicates that fewer nonrecommended drugs (including opioids) were prescribed in Texas after the state implemented a drug formulary (TDI, Texas Workers’ Compensation Research and Evaluation Group, 2013). Evidence from the 2018 formulary in California indicates a decrease in the share of prescriptions for drugs that were not exempt from prospective utilization review in the formulary, with opioid prescriptions contributing more than half of the decrease (Hayes and Swedlow, 2019).

States have also implemented a number of other policy interventions aimed at limiting excessive prescribing and opioid abuse. These include regulating pain clinics and limiting the dispensing of controlled substances by physicians. Prior studies suggest that opioid prescriptions dispensed to injured workers decreased in Florida after 2011 legislation regulating pain clinics and banning physician dispensing of Schedule II and III opioids, and in Kentucky after 2012 rule changes limiting dispensing of Schedule II and III opioids (Thumula, 2014, 2017).

\footnotetext{
${ }^{14}$ For example, ACOEM guidelines recommend limiting opioid prescriptions to two weeks. When treating acute pain, CDC guidelines suggest that physicians should prescribe the lowest effective dose, that 3 days of opioid prescriptions may often be enough, and that physicians should rarely need to prescribe more than 7 days of opioids (CDC, 2016). The guidelines are not mandatory and leave decisions to the discretion of the provider.

${ }^{15}$ For example, the 2014 update of the ACOEM guidelines sets the MME dose at 50 milligrams.
} 
We provide several tests that demonstrate that these additional policies are unlikely to substantially influence our estimated effects of PDMPs and initial prescribing limits. First, we examined changes in outcomes prior to the policy changes we study to ensure that states that did and did not introduce the policies that we examine had similar evolution of outcomes prior to these two policy interventions. Second, we show (in the online appendix) that some additional policies by and large did not influence the outcomes we study, and that including them in our analysis does not alter the estimated effects of PDMPs and initial prescribing limits.

The policies outlined above do not cover all of the initiatives designed to curb opioid overuse and abuse. Some states implemented mandatory provider education for safe opioid prescribing, conducted educational campaigns for patients about the dangers of opioid prescribing, and required written pain management plans for patients who received opioids. States may also require continuing medical education on opioid prescribing and chronic pain management as a requirement for license renewal. Evidence about the effects of these interventions is quite limited, with earlier studies showing that most states did not mandate provider education (Davis and Carr, 2016). A review of the evidence in BenShalom et al. (2020) found only a few studies on the effects of provider education with strong research designs; randomized control trials showed little effect of provider education on opioid prescribing (Pasquale et al., 2017). Likewise, Ben-Shalom et al. (2020) found only weak evidence of effects of patient education on opioid use. The changing prescribing practices may also be driven by multifaceted interventions combining multiple practices for safe prescribing introduced by health systems as well as some states. These interventions often included implementation of guidelines, provider training, patient outreach, and improved monitoring and communications between providers and patients (for a review, see Ben-Shalom et al., 2020). In our analysis, we examine the sensitivity of our estimates to adding controls for various treatment guidelines, but we did not collect information on provider or patient education requirements. At the federal level, changes in the CSA or REMS programs may have influenced opioid prescribing across all states. We address concerns about these federal regulations when 
we discuss our empirical approach. ${ }^{16}$

\section{Data and Methods}

Our analysis sample was derived from payment information on workers' compensation claimsthe Workers Compensation Research Institute (WCRI) Detailed Benchmark/Evaluation (DBE) database. The DBE covers claims from national and regional insurers (including residual market carriers), state funds, and self-insured employers (from their third-party administrators). Since our main objective is to examine utilization of care (including opioid prescriptions) and duration of disability, we extracted data on filled prescriptions, medical services that were provided, and temporary disability benefits made within 12 months after an injury. ${ }^{17}$ The DBE includes detailed prescription transaction data and medical billing data that were collected from workers' compensation payors and their bill review and pharmacy benefit vendors. The data available for each prescription identify the specific medication prescribed based on National Drug Code (NDC), the date on which the prescription was filled, amounts charged and paid, the number of pills (for orally-administered opioids), and the strength of the medication in milligrams. ${ }^{18}$ The data on other medical services include information on procedure type and date. The analysis includes information for workers injured between October 1, 2009, and March 31, 2018, in the 33 states covered in the DBE database. ${ }^{19}$ These states represent 85 percent of benefits paid in 2017 (Weiss et al., 2019).

We focus our analysis on all claims and on the subset of claims with opioids (when studying characteristics of the opioid prescriptions). Many studies of workers' compensation outcomes focus on

\footnotetext{
${ }^{16}$ States also adopted policies to try to reduce opioid overdoses and deaths. These include Good Samaritan laws that provide immunity from criminal prosecution for individuals helping themselves or others experiencing an overdose, laws providing support for naloxone access, and syringe exchange programs. Since we do not examine potential overdoses and death, these policies are also outside the scope of our analysis.

${ }^{17}$ Our results are robust to using data that instead captures care provided within 6 months after an injury (see the online appendix). The different observation windows after an injury reflect different "maturities" of the data that may be available. We cannot use longer maturities because a good deal of the policy variation we study is recent.

${ }^{18}$ We linked the prescription transactions to the Medi-Span ${ }^{\circledR}$ data by NDCs to identify the type of medication (e.g., drug name, therapeutic class, formulation, and strength). We used the classification scheme provided by MediSpan ${ }^{\circledR}$ 's Generic Product Identifier (GPI) to assign drugs into different therapeutic drug groups.

${ }^{19}$ The states are Alabama, Arkansas, Arizona, California, Connecticut, Delaware, Florida, Georgia, Hawaii, Illinois, Indiana, Iowa, Kansas, Kentucky, Louisiana, Maryland, Massachusetts, Michigan, Minnesota, Mississippi, Missouri, Nevada, New Jersey, New Mexico, New York, North Carolina, Oklahoma, Pennsylvania, South Carolina, Tennessee, Texas, Virginia, and Wisconsin.
} 
claims with more than 7 days of lost work time, as it is these claims, in general, that entail indemnity benefits and receive most of the medical care ${ }^{20}$ However, we do not want to condition on the number of days of lost work time because it may be influenced by opioid prescribing behavior, leading to biased estimates of the policy effect. ${ }^{21}$

We estimate the effects of state policies—-must-access PDMPs and initial prescribing limits—by estimating regression models that compare how outcomes changed in states that adopted the policies (“treated states”) relative to states that did not (“control states”), accounting for other factors that could have affected these outcomes. While the federal efforts to combat opioid overuse likely contributed to the decline in opioid utilization across all states, our estimates reflect effects of state policies above and beyond the effects of the federal policy changes that should have affected every state. ${ }^{22}$

For our results to be valid, we have to believe that there were no other factors that led to divergent paths of the outcomes in the treated and control states. We ensure this, in part, by including in our statistical models control variables for other determinants of differential outcomes by state. These include industry composition, ${ }^{23}$ average county-level unemployment rates, ${ }^{24}$ average county-level median household income, average county-level percentage disabled, and average county-level percentage without health insurance. ${ }^{25}$ The county-level information was added to the individual claim level data and then aggregated to the state level. ${ }^{26}$ These controls address concerns about a changing mix of industries in treatment versus control states, changes in the access to employer-provided medical insurance over time

\footnotetext{
${ }^{20}$ Among claims that did not have more than 7 days of lost time, 9.1 percent had prescriptions for opioids in our sample.

${ }^{21}$ For example, suppose a particular policy both reduced opioid prescribing and decreased the days of lost work time. Then, if we restricted the analysis to claims with more than 7 days of lost work time, we might fail to detect the decline in opioid prescribing.

${ }^{22}$ Therefore, our estimates likely understate the combined effects of federal and state policy changes.

${ }^{23}$ We control for the shares in high-risk services, low-risk services, clerical/professional occupations (regardless of industry), manufacturing, construction, trade, and other industries, in the sample of injured workers that we use.

${ }^{24}$ We use the U.S. Bureau of Labor Statistics' Local Area Unemployment Statistics (LAUS); see https://www.bls.gov/lau/.

${ }^{25}$ These measures were derived from the U.S. Health Resources and Services Administration's Area Health Resources Files (AHRF); see https://data.hrsa.gov/data/download/.

${ }^{26}$ We match county-level information to our data using workers' county of residence (derived from workers' residence zip code information). When the residence location information is not available, we use location of the most frequent visit for health evaluation and management services, location of the accident, or location of the employer.
} 
in different states, etc. We also control generally for other state-specific factors that do not change over time and time effects that are common across states, by including fixed state effects and fixed quarter effects (for each unique quarter in the data). ${ }^{27}$

We also pay attention to the possibility that there are unmeasured factors that drive additional variation in outcomes across states. Since they are unmeasured, we cannot directly incorporate them into our models. However, we check whether, prior to the policy change, trends in outcomes in the treated and control states, which may be attributable to unmeasured influences on the outcomes, are the same or not (i.e., do the "parallel trends” hold?). If there are parallel trends, we are more confident that the control states provide valid predictions for what would have happened in the treated states had the policy changes not occurred; if parallel trends are violated, we have less confidence in this approach and have to consider modifications of our statistical model that control for these trend differences to provide more reliable evidence, if possible. In fact, this part of our analysis turns out to be quite important for estimating the effects of initial prescribing limits.

Above, we explained that we focus on two types of state policy changes intended to restrict opioid prescribing: must-access PDMPs, and limits on the initial supply of opioids. We focus on these two policies mainly because there are too few instances of the other types of opioid-related state policy changes to be able to reliably estimate their effects. However, we also show (in the online appendix) that there is no clear evidence that these other policies mattered, and we show that excluding them from our analysis does not materially impact our estimated effects of PDMPs and initial prescribing limits.

We aggregate data to cells defined by state and calendar quarter of injury, and weight by the number of observations within each cell. These cells provide average outcomes of those who were injured in a quarter and reflect experience up to 12 months postinjury. Our analysis also accounts for partial exposure to policies during the 12 months, which can occur when a policy was implemented during the 12-month period after an injury date and hence would have affected only a subset of the services

\footnotetext{
27 The former account for differences across states in time-invariant workers' compensation policies, and other measures that are constant over time. The latter reflect factors that are changing across all states at the same time, such as federal policies.
} 
provided. For example, if the policy was implemented 3 months after an injury, then the medical services for the injury provided after month 3 would be affected by the policy, while services provided in the first 3 months would not be subject to the policy. That is, the effects up to any point in the 12 months since the injury occurred may reflect not simply the policy in effect at that point but the cumulative effects of the policy up to that point—which can depend on how long the policy has been in effect. Since we estimate regressions with single variables capturing each of the two policy changes-PDMPs and prescribing limits—we capture the partial exposure of those injured prior to policy changes in a richer manner than simply using a dummy variable for whether a policy was in effect. In particular, we define the policy variable as 1 if it was in effect for all 12 months of postinjury exposure (like a standard dummy variable), 0.75 if it was in effect for 3 of the 4 quarters of postinjury exposure, 0.5 if it was in effect for 2 of the 4 quarters of postinjury exposure, and 0.25 if it was in effect for only the last quarter of the 4 quarters of postinjury exposure. ${ }^{28}$

\section{Results for Opioid Prescribing}

We begin with event-study estimates of the effects of opioid prescription policies. We plot results from regressions that estimate the differential evolution of the outcomes we study in states that did and did not adopt these policies, by quarter. (For the states that did not adopt these policies, the regression model allows for different outcomes by quarter, via the inclusion of quarter indicator variables.)

For each of the two policies, we estimate a model of the form:

(1) $y_{s t}=\sum_{k=2}^{7,8+}\left\{\gamma_{-k} \times P O L_{s, t-k}\right\}+\sum_{j=0}^{5,6+}\left\{\delta_{j} \times P O L_{s, t+j}\right\}+x_{s t}+\gamma_{s}+\eta_{t}+\varepsilon_{s t}$.

$\gamma_{s}$ are state fixed effects, and $\eta_{t}$ are fixed effects for each unique quarter. The reference period is $t=-1$. The $P O L$ terms are separate dummy variables equal to one in states where the policy was implemented, with separate variables for $t=-2,-3, \ldots,-7$, and -8 or less, and for $t=0,1, \ldots, 5$, and 6

\footnotetext{
${ }^{28}$ We also show in the online appendix that the results are not sensitive to using partial exposure weights that are more data-driven, reflecting the cumulative number of opioid prescriptions in different parts of the 12 months postinjury experience, rather than partial exposure weights based simply on the proportion of time "exposed" to the policy. This addresses concerns that the medical services we study in the 12 months since the injury do not necessarily accumulate linearly over time, but may instead, for example, be more heavily concentrated soon after the injury (which is in fact what we see in the analysis in the online appendix).
} 
or more. Thus, the parameters $\gamma_{-k}$ capture pre-treatment differences and the parameters $\delta_{j}$ capture the evolution of post-treatment differences. Note that $t=0$ is the first quarter of partial exposure to the policy, and $t=3$ is the first quarter of full exposure. ${ }^{29}$ We estimate equation (1) for each policy separately.

These estimates address a few key questions. First, the estimates prior to policy adoption tell us whether there were different prior trends in the treated and control states. If there were, the parallel trends assumption that underlies the identification strategy would not hold, implying that the post-treatment estimates should not be strongly interpreted as causal, at least without correcting for these prior trends (if possible). Second, the estimates after policy adoption are informative about the evolution of the policy effect. One thing we expect is that the effects of the policies should emerge over the first year of adoption, depending on how many months of the 12-month maturity period the policy is in effect. Third, the estimates after policy adoption tell us about the dynamics of the effects of the policy changes-for example, whether the effects of the policy fade sometime after adoption. However, we do not have very much post-adoption evidence given how late the policies changed in some states, and hence our estimates become noisier (from a statistical perspective) the farther we look post-policy adoption. ${ }^{30}$

The estimates of equation (1), and 95 percent confidence intervals, are displayed in Figure 2, graphed in black; we return to the estimates graphed in gray below. The height of the plotted points is the estimated difference between treated and control states, at the leads and lags (by quarter) indicated on the horizontal axis. The vertical lines through the plotted points are 95 percent confidence intervals.

The event-study estimates for PDMPs are on the left-hand side. Looking first at the pre-trends (estimates before quarter 0 ), there is very little evidence of violation of the parallel trends assumption. ${ }^{31}$ There is one instance (for MME for all claims, in the top row) where there is some hint of a downward trend prior to the policy change, but the trend is not present in the few quarters before the change.

Looking at the treatment effect for PDMPs (beginning with quarter zero), for all outcomes we see

\footnotetext{
${ }^{29}$ In the event study estimates, $P O L$ is simply a dummy variable; in the regression estimates that follow, it is defined using the partial exposure weights described just above.

${ }^{30}$ These longer-term estimates also become less representative of all treated states.

${ }^{31}$ We present statistical tests for pre-trends in the online appendix; these largely parallel the conclusions we draw in this discussion.
} 
evidence of declines in the opioid prescribing measure post-treatment. As we would expect, in the quarters of partial exposure there is less evidence of an effect. And for four of the five outcomes (MME for all claims, number of prescriptions for all claims, MME for claims with opioids, and number of prescriptions for claims with opioids), there is evidence of a persistent and perhaps even continuing decline after full exposure to the policy change (indicated by the second vertical line).

The estimates of equation (1) for prescribing limits are on the right-hand side of Figure 2, also graphed in black. ${ }^{32}$ Looking first at the pre-trends, two of the three outcomes for all claims - any opioids, and the number of opioid prescriptions for all claims-appear problematic, with both trending up before the treatment. Moreover, the trend largely continues, in linear fashion, after the treatment. This implies that estimated regression models that do not take account of these trends are likely to generate spurious evidence of a positive effect of prescribing limits on whether opioids are prescribed, whereas models that account for these trends appear unlikely to generate evidence of an effect. For the claims with opioids, there are no apparent pre-trends. Moreover, it appears that there may be a negative effect on MME (for claims with opioids) from initial prescribing limits. ${ }^{33}$

While the estimates in Figure 2 are useful for describing the path of changes in opioid prescribing that are associated with the policy changes we study, they do use the data rather inefficiently because, effectively, separate treatment effects are estimated for each quarter. We therefore build on what we have learned so far to construct regression models that capture what our detailed analyses have shown, but in a single parameter that measures the effect of the policy change, using a more-standard two-way fixed effects model (with some modifications).

\footnotetext{
${ }^{32}$ Note that although only three states had these laws by the end of 2016, we nonetheless have seven states contributing to the $\geq 6$ quarter estimates for the opioid limit policies (although three of those states contribute only one quarter of data). Quarter 0 indicates the start of the partial exposure to the policies (only the last quarter of the care within a year post injury is subject to the policy), and quarter 3 indicates full exposure to policy (i.e., covering four quarters) for all care provided for the injury.

${ }^{33}$ The online appendix also includes descriptive graphs on the effects of policy changes state by state. These graphs are useful in providing evidence on how heterogeneous effects might be across states, which is relevant to the question of how meaningful an estimate of the "average" effect of an opioid prescription policy across states is. We read the graphs as indicating that there is not too much heterogeneity, although there are some exceptions.
} 
Before doing so, however, we explore the potential implications for our analysis of issues raised in the recent econometric literature on two-way fixed effects (TWFE) models. The key issue is whether TWFE estimates are substantially biased relative to what we would get from one of the new estimators to address potential biases when treatment is staggered and treatment effects are heterogenous (Callaway and Sant'Anna, 2021; Goodman-Bacon, 2021; Jakiela, 2021; Sun and Abraham, 2021; Wooldridge, 2021). Heterogeneous treatment effects could arise because, for example, states hit hardest by the opioid pandemic may have adopted PDMPs earlier, and could have—-because of the more severe crisis— enforced them more rigorously. Dynamic heterogeneity could have arisen if later policies had less impact because of rising awareness or caution in prescribing opioids with increased attention paid to the pandemic, or because effects simply change over time since a policy is implemented.

We have explored a number of approaches suggested by this literature for assessing the potential bias. First, we assess the robustness of the event-study estimates in Figure 2 to using the Sun and Abraham estimator. ${ }^{34}$ Second, a relatively large number of the states that adopted initial prescribing limits did so late in the sample, creating a greater potential for bias when earlier adopters are compared to them rather than to never-treated states (Goodman-Bacon, 2021). We thus also report event-study estimates for initial prescribing limits (for which there is a good deal of late adoption), excluding the states that adopted these limits late. The Sun and Abraham estimates for the full sample are graphed in grey in Figure 2; they are largely indistinguishable from the standard event-study estimates. Figure 3 then provides both sets of estimates for the sample excluding the late adopted. The estimates are very similar to each other, and very similar to the estimates in Figure 2. Given this evidence, we go on to report our modified two-way fixed effects estimates using the full data set.

In particular, we estimate specifications of the form:

(2) $y_{s t}=\beta \times P O L_{s t}+x_{s t}+\gamma_{s}+\eta_{t}+\varepsilon_{s t}$

\footnotetext{
${ }^{34}$ We feature the Sun and Abraham estimates following the advice of Wooldridge (2021, pp. 68-9), who recommends Sun and Abraham types of methods that continue to use simple regression models. (However, we have also verified that the estimates are similar using the Callaway and Sant'Anna approach - estimates that are available upon request.)
} 
Note that in this model $P O L_{s t}$ equals $0.25,0.5$, and 0.75 in the first three quarters after implementation of the policy, and 1 thereafter.

We estimate regressions with separate variables capturing the two policy changes_-PDMPs and prescribing limits. We vary our empirical specifications to address concerns about divergent trends in outcomes before policies were introduced (as highlighted in some of the panels in Figure 2 for the effects of initial prescribing limits). In particular, we show results that account for different state-specific trends in outcomes, and, given the difficulty of estimating a causal effect of initial prescribing limits on outcomes for the all claims sample, we also highlight results from the specifications that focus only on PDMPs.

Table 2 reports our regression estimates for the five outcomes we use to characterize opioid prescribing. Columns (1)-(3) report estimates (for all claims) for MME, for whether any opioids were prescribed (the proportion of claims with opioids prescribed), and for the number of prescriptions. Columns (4) and (5) report results for MME and the number of prescriptions for the subset of claims with opioid prescriptions. The first rows of each panel report the regression coefficients that measure the impact of the policy changes on the outcome. We also show the sample means of the dependent variables and the percentage change in the outcomes implied by applying the estimated impact to the sample mean.

We begin, in Panel A, with estimates that simply include the timing-adjusted dummy variables to estimate the effects of the introduction of PDMPs and initial prescribing limits, without accounting for the pre-trends we saw in Figure 2 in relation to initial prescribing limits. Looking first at PDMPs, we find that the policy reduced MME for all claims by 20.1, or 12 percent (column (1)), and MME for claims with opioid prescriptions by 131.1—a similar amount in percentage terms (12 percent) because MME conditional on prescriptions excludes many zeros (column (4)); these estimates are significant at the 5 or 1 percent level. We do not find an effect of PDMPs on whether opioids were prescribed or, for all claims, on the number of prescriptions (columns (2) and (3)); in both cases, the estimates are not significantly different from zero, and the estimated effects are small in percentage terms. Among claims with opioid prescriptions, however, we find that PDMPs reduced the number of prescriptions by 0.15 , implying a 5 
percent reduction, and the estimated effect is strongly statistically significant (at the 1 percent level). These effects of PDMPs are consistent with what we would expect given the goal of the policy. We should expect PDMPs to reduce the number of prescriptions, and we would anticipate this effect to be concentrated among those with prescriptions.

Next, consider the evidence on limits on initial opioid prescriptions. The evidence for all claims points to positive effects on whether opioids were prescribed and on the number of opioid prescriptionseffects that are large (15 percent and 18 percent, respectively) and statistically significant. However, these are the two outcomes for which the event-study graphs in Figure 2 suggested we could find a spurious positive effect if we did not account for the trends in the opioid measures that prevailed even before the policy changes. For that reason, these positive estimates are unreliable.

The evidence on claims with opioids is similar to that for PDMPs. Initial prescription limits appear to reduce MME (a 19 percent reduction), although the effect on the number of prescriptions is small. Figure 2 did not indicate problems with interpreting the estimates for the claims with opioids as problematic based on pre-trends, so we view this evidence as more plausibly causal. Presumably, this evidence reflects initial prescribing limits reducing opioid amounts among those who are prescribed opioids, consistent with how the policies are structured.

Panel B of Table 2 reports estimates of regression models that add state-specific trends. The evidence from Figure 2 suggests these are important for the evidence on the effects of initial prescribing limits on any opioids and on the number of prescriptions for all claims. Indeed, as Panel B shows, the estimated effects of initial prescribing limits on these outcomes now become small and statistically insignificant (columns (2) and (3)). The estimated effects of PDMPs are qualitatively similar but smaller. $^{35}$

The estimates for the claims with opioids, in columns (4) and (5), are qualitatively similar with the state-specific trends included. We still find negative and significant effects of PDMPs on MME and

\footnotetext{
${ }^{35}$ We estimated the event-study models in Figure 2, for this outcome, with state-specific trends added to the models. These diminished the evidence of pre-trends in the periods immediately preceding the treatment. (These results are available upon request.)
} 
on the number of opioid prescriptions, although the estimates are a little smaller. The estimated negative effect of initial prescribing limits on MME for claims with opioids also remains negative and statistically significant, although it is about one-third smaller (an 11 percent reduction, versus 19 percent in Panel A).

Finally, while the state-specific trends are needed to obtain plausibly causal estimates of the effects of initial prescribing limits on the outcomes for all claims, models with these included do not necessarily recover the most reliable estimates of the effects of PDMPs, since there are no prior trends in the outcomes in relation to the adoption of PDMPs. When state-specific trends are included but not needed, they can mask or distort the actual treatment effect, especially when the treatment effect grows over time (Meer and West, 2016). Thus, in Panel C we report estimates of the models without the trends, but including only the PDMP policy variable. These estimates are quite similar to those in Panel A, indicating that PDMPs reduce MME for all claims, and reduce MME and the number of prescriptions for claims with opioids.

We next turn to the analysis of outcomes for specific injury groups, and (in the following subsections) to estimated effects on other outcomes, including alternative treatments. Given what we have found and reported regarding the association between policy adoption, outcomes, and pre-trends, going forward when we estimate the effects on outcomes for all claims, we simply omit initial prescribing limits and focus only on the effects of PDMPs (like in Panel C of Table 2). We do so because our preceding analysis suggests there is no causal effect of initial prescribing limits, but if we include the initial prescribing limits variable we have to include state-specific trends, which can create bias toward zero in the estimated effects of PDMPs. On the other hand, we have shown that omitting the initial prescribing limits does not alter the estimated effects of PDMPs. When we estimate effects on outcomes for claims with opioids, however, we do not need the state-specific trends; hence, we use the models in Panel A that include both policy variables_-for PDMPs and initial prescribing limits.

In Table 3, we look at evidence for different injury groups that reflect common conditions for workers with injuries. ${ }^{36}$ Among the injuries we examine, workers with neurologic spine pain had the

\footnotetext{
36 The group of “other injuries” includes heterogeneous injuries that do not fit in main groups specified in Table 3.
} 
highest likelihood of receiving opioids, and had the highest average MME per claim (see the means in Table 3). We estimate the effects of PDMPs on MME per claim, whether any opioids were prescribed, and number of opioid prescriptions for all claims (Panels A through C); and we estimate the effects of PDMPs and initial prescribing limits on MME and number of opioid prescriptions for the subsample of claims with opioids (Panels D and E).

As shown in Table 3, the effect of must-access PDMPs on MME per claim for all claims as well as on MME per claim with opioids is a statistically significant reduction (at the 10 percent level or less) for neurologic spine pain, spine sprains and strains, and other sprains and strains cases; and we find a similar reduction for MME for claims with opioids for inflammations. The common evidence of the effects of must-access PDMPs on MME per claim — whether or not we condition on an opioids prescription—is for neurologic spine pain, spine sprains and strains, and other sprains and strains cases. For these injury groups, the estimated effects are sizable-10 to 21 percent in Panel A, and 11 to 21 percent in Panel D. (For the other injury groups, the estimated effects are smaller and statistically insignificant.) Furthermore, we see little shift in the percentage of claims with opioids (Panel B) and a decrease in the number of opioid prescriptions among claims with prescriptions (Panel E) due to PDMPs. This evidence suggests that PDMPs lead to fewer cases with large numbers of prescriptions for neurologic spine pain, spine sprains and strains, other sprains and strains cases, and inflammations (some of which have quite high average MME).

The estimated impact of initial prescribing limits on MME for claims with opioids is nearly uniformly negative across injuries, and statistically significant for five of the eight injury categories; the estimates are sizable for most injury categories (Panel D). Negative effects of initial prescribing limits on MME per claim with opioids are apparent for fractures, inflammations, neurologic spine pain, other sprains and strains, and the group of "other injuries.” Note that the effects of initial opioid limits on number of opioid prescriptions (among those with opioids) differed across injury groups—an increase for lacerations and contusions, a decrease for other sprains and strains, and little change for most other injuries (Panel E). Thus, in comparing the results for must-access PDMPs and initial prescribing limits, 
for claims with opioids, there is common evidence of reductions in MME for inflammations, neurologic spine pain, other sprains and strains, and other injuries; the evidence for number of prescriptions is less consistent across injury groups.

In general, then, we find that must-access PDMPs and initial prescribing limits affected the intensive margin (although we should be a little more cautious about results for initial prescribing limits in light of prior trends). These responses seems natural. For example, PDMPs ought to provide information about prescriptions already provided to a patient and hence potentially reduce excess prescribing, ${ }^{37}$ whereas it is not clear why there should be a direct effect on the extensive margin.

\section{Results for Problematic Opioid Prescribing}

Policies to reduce opioid use were adopted in large part to reduce problematic prescribing since multiple concurrent prescriptions may indicate excessive opioid use or opioid abuse. To assess the effectiveness of the policies on this dimension, we examine the effects of PDMPs and limits on initial opioid prescriptions on the proportion of claims with opioids with three alternative measures of problematic prescriptions: longer-term opioid prescribing (prescriptions within the first three months of an injury and three or more filled prescriptions between the 7th and 12th months after an injury); more than 90 days of opioids prescribed; and a morphine equivalent daily dose (MED) exceeding 120 mg. ${ }^{38}$ While it is difficult to distinguish between appropriate and inappropriate use of opioids, the metrics presented here reflect higher quantities of opioids being prescribed, which may indicate potential misuse or at least dangerous use that may lead to addiction (for a review of evidence see CDC, 2016). ${ }^{39}$

We report the regression results for all claims and for all claims with opioids in Table 4. We find strong evidence that PDMPs reduced longer-term prescribing for claims with opioids; the estimate

\footnotetext{
${ }^{37}$ Alpert et al. (2020) find this effect via an information channel, but also via what they term "hassle costs," in studying Kentucky’s PDMP. Buchmueller et al. (2020) study the same policy, and find evidence of an extensive margin response.

${ }^{38}$ As discussed above, we only estimate the effects of initial prescribing limits for the claims with opioids. Examination of event-study graphs similar to those in Figure 2, for the problematic opioid prescribing outcomes, revealed similar concerns about pre-trends in estimating the effects of initial prescribing limits on the outcomes for all claims (results available upon request).

${ }^{39}$ Similar metrics were used in prior studies along with measures indicating multiple prescribers and multiple pharmacies where the opioids where dispensed (Buchmueller and Corey, 2018; Sacks et al., 2019).
} 
implies a 12 percent reduction, and is statistically significant. None of the other estimates in the table are statistically significant, and except for the estimated effect on daily dose greater than $120 \mathrm{mg}$, for all claims, the other estimates are small in percentage terms. ${ }^{40}$

Table 5 reports estimates of the models for problematic use estimated by injury group. The results in Table 5 for all claims, in the top three panels, are almost all statistically insignificant, with one exception.

However, when we look at the results for claims with opioids (in the bottom three panels), we see more evidence that PDMPs reduce problematic use. This evidence is most pronounced for longer-term opioid prescribing — the same measure for which we found that PDMPs reduce use across all injuries combined (in Table 4). Here, we see a similar reduction in longer-term prescribing for inflammations, spine sprains and strains, other sprains and strains, and other injuries. There is also a negative effect for neurologic spine pain, but it is not statistically significant. In addition, there are a few negative and significant estimates for the other two measures of problematic prescribing, in one case (for daily dose greater than $120 \mathrm{mg}$ ) for neurologic spine pain. We read the evidence in Table 5 as suggesting that PDMPs had some impact in reducing problematic opioid prescribing among claims with opioids. The weaker evidence in the top three panels — for all claims - is likely attributable to the fact that PDMPs do not restrict whether one gets opioids or not. Rather, they restrict the amount of opioids for those who are prescribed opioids.

\section{Results for Substitution toward Other Kinds of Medical Care}

The evidence to here suggests that PDMPs reduce opioid prescribing, amounts prescribed, and longer-term or problematic prescribing. These results for PDMPs raise the question of whether the opioid reductions lead health care providers to substitute other kinds of care. If (as the preceding evidence suggests) policies reduce access to opioid therapies, workers may seek other treatments to deal with their

\footnotetext{
${ }^{40}$ We also estimated the same specifications as in Table 2 for these outcomes. The results are generally similar, with a robust finding for the effects of must-access PDMPs on longer-term opioid prescribing. There are some other significant results for the models when we introduce state-specific trends. But absent evidence of pre-trends in these specifications, we do not think the results with the trends included should be viewed as reliably, since (as shown in, e.g., Meer and West, JHR, 2016) trends can get confounded with causal effects. These results are available upon request.
} 
pain. This care may include non-opioid pain medications, interventional pain management, and physical therapy.

Interventional pain management treatment includes discography and disc decompression, electrical stimulation implants, epidural procedures, pump implants, trigger point injections, vertebra augmentation procedures, other injections, and nerve blocks. These are typically done in doctors' offices and are more invasive than prescriptions. Active physical medicine services are defined as strength training and/or conditioning exercises performed by patients under the direction of a physical therapist. Non-opioid pain medications include non-opioid analgesics (such as nonsteroidal anti-inflammatory drugs and acetaminophen) and adjuvant analgesics (such as corticosteroids, anticonvulsants, antidepressants, and dermatological treatments); this definition follows prior studies examining prevalence of pain medications in workers' compensation system (Thumula et al., 2019). This category of care may also include chiropractic care as well as acupuncture, although this care is not widespread in some of the states we study.

Note that we focus on types of care that are often recommended in treatment guidelines as potential substitutes for opioid prescriptions when dealing with acute and chronic pain (see Thumula et al., 2019). Whether the emphasis is on using non-opioid pain medications or non-pharmacological treatments, the recommendation is to approach opioid-based treatment with caution. ${ }^{41}$

There are different possible interpretations of evidence on the effects of policies restricting opioid prescribing on alternative treatments. A decrease in opioid prescribing coupled with an increase in potential substitutes for opioids may indicate that workers still had pain but were able to find other treatment that is recommended in guidelines. While workers avoided exposure to potentially addictive substances, we do not know if the alternative approaches to pain led to improved recovery or outcomes. There is also ambiguity in interpreting results indicating a decrease in opioid utilization without a

\footnotetext{
${ }^{41}$ And as noted earlier, Mallatt (2022) studies effects of opioids-reduction policies (and the reformulation of OxyContin) on substitution towards heroin, based on crime data on heroin possession and dealing, finding evidence that optional-access PDMPs generated substitution towards heroin; the evidence for must-access PDMPs points in the same direction, but is statistically weaker. Of course this channel of influence of opioid policies may be more related to addiction than to pain management.
} 
corresponding increase in substitute care. One possibility is that opioid prescriptions might have been unnecessary. For instance, workers might have received opioid prescriptions for multiple days supplied but only took a few pills and did not have a need for other prescriptions. Alternatively, workers may have still had pain, but faced issues accessing appropriate providers to receive alternative care (e.g., pain specialists for pain management were not available, or they reached limits on physical therapy visits). Since we do not have detailed medical information to determine whether any of the opioid prescriptions were unnecessary, we limit this discussion to highlighting different patterns of results that we see. We do think, though, that information on management of pain in response to changes in patterns of treatment is an important research need.

As a preliminary analysis, we constructed event-study graphs to examine pre-policy trends for these measures across injury groups. For most policy measure and injury group combinations, we found little evidence to contradict the parallel trends assumption. We found a few cases where event-study graphs indicated that differences in trends may have emerged prior to the policy implementation, mostly for limits on initial opioid prescribing, which—for this reason, paralleling what we did earlier—we do not consider here, instead focusing only on PDMPs. For the analysis of PDMPs, we found two instances where event-study graphs indicate that trends may have emerged before the policy. For these two cases (the effects of PDMPs on the number of non-opioid pain medications and on the percentage with active physical medicine services for lacerations and contusions), we estimate the models with state-specific time trends. $^{42}$

For this analysis, presented in Table 6, we report results by injury type. Results by injury type are more meaningful because different types of health care are more appropriate for one kind of injury than another. For example, the nature of treatment is well determined for someone with a fracture, and there may be little flexibility to choose different treatment approaches or types of care. For soft-tissue injuries or for non-specific low-back injuries, the treatment approaches vary much more widely across providers and across parts of the country, so there is more flexibility in treatment approaches (e.g., Yee et al., 2015).

\footnotetext{
${ }^{42}$ These event-study graphs are available upon request.
} 
This analysis is done for all claims, and not for claims with opioids, since the question we consider is what kind of care is provided as a potential alternative to opioids.

We begin, in the first row, by repeating the estimated effects on MME for all claims, to provide a contrast with alternative treatments. The key question in this table is whether PDMPs lead to substitution toward other kinds of medical care. There is some evidence of this for particular injury types, but not for others. For spine sprains and strains, we see little evidence of substitution to other types of care. While the MME decreased, the use of other types of care did not change. For other sprains and strains, we see some evidence of an increase in the number of non-opioid pain medications. The most consistent evidence of substitution is for lacerations and contusions, where PDMPs lead to significant increases in non-opioid pain prescriptions, and in interventional pain management services being provided and the number of visits for these services. Although the evidence for lacerations and contusions indicates that PDMPs generate substitution toward different types of alternative care, we saw little change in opioid prescribing for this group of injuries (and the amount of opioids prescribed for these injuries is the lowest of the injuries we examine). To be clear, though, the absolute sizes of the effects are very small (changes of less than a one-quarter of one percentage point).

There is also some evidence of substitution for neurologic spine pain in response to PDMPs. For this group of injuries, we observe a 13 percent decrease in the MME, a 14 percent increase in the number of non-opioid pain medication prescriptions, and increases in interventional pain management services being provided and the number of visits for these services. The evidence on the management of neurologic spine pain is interesting because, among the injury groups we consider, it has the highest incidence of opioid prescriptions and the highest MME.

\section{Results for Duration of Temporary Disability}

Finally, in Savych et al. (2019), we found that longer-term opioid prescribing for low-back injuries increased the duration of temporary disability benefits (which are paid, in most states, for claims with more than 7 days of lost work time). The study also found little effect of receiving any opioid prescription on the duration of temporary disability benefits. Our evidence was not based on simple 
associations (controlling for worker and injury characteristics) between longer-term prescribing and duration, which we would expect to be positive because longer-term prescribing is more likely for more severe injuries_ — even on dimensions we cannot measure. Rather, we isolated the effect of exogenous variation in longer-term prescribing based on local prescribing patterns.

In this study, given that we find some evidence that PDMPs reduce longer-term opioid prescribing, we revisit the question of longer-term prescribing and the duration of temporary disability. Here, we look at a different source of exogenous variation in longer-term prescribing — namely, the adoption of PDMPs. Given that the duration of disability outcome is potentially quite different from the outcomes studied in earlier tables, in Table 7 we repeat the three kinds of analyses reported in Table 2: estimates of the effects of both PDMPs and initial prescribing limits with and without state-specific trends, and then estimates of the effects of PDMPs only, without these trends.

A comparison of Panels A and B shows that, as in Table 2, the models without state-specific time trends generate spurious evidence of a positive effect of initial prescribing limits. With the trends included, there is no statistical evidence of an effect of these limits, although the estimates are not very precise and hence do not necessarily rule out non-trivial reductions. ${ }^{43}$ Regardless of the specification, there is no statistically significant evidence of effects of PDMPs, and the confidence intervals cover a wide range of possible estimates, including substantial positive or negative effects. ${ }^{44}$

This may not be surprising since the primary goal of PDMP policies is to limit excessive prescribing and simultaneous prescribing by multiple providers. In contrast, the key finding in Savych et al. (2019) was that longer-term opioid prescribing led to increases in the duration of temporary disability benefits, while, in this paper, we do not find an effect of PDMPs on longer-term opioid prescribing. ${ }^{45}$

\footnotetext{
${ }^{43}$ These findings are reflected in event-study graphs paralleling those in Figure 2 . There is evidence of pre-trends in the direction of increasing duration for states adopting initial prescribing limits, necessitating the inclusion of statespecific trends to obtain reliable evidence on the effects of these limits. These event-study graphs are available upon request.

${ }^{44}$ We also estimated these same three sets of models for each of the injury types (as in, e.g., Table 6). The results, available upon request, almost always indicate no significant differences. A smattering of significant coefficients is expected because of the large number of estimated coefficients.

${ }^{45}$ The relevant result is in column (1) of Table 4. There is some evidence that PDMPs reduced longer-term prescribing for those with opioids, but the analysis in Savych et al. (2019) did not condition on receiving opioids.
} 
Thus, the effects of these policies on duration of disability may be a secondary effect at best.

\section{Conclusions}

We provide evidence on the effects of must-access PDMPs and regulations limiting the duration of initial opioid prescriptions on utilization of opioids, utilization of care that may be a substitute for opioid prescriptions, and duration of temporary disability benefits. We find that must-access PDMPs contributed to a decline in opioid utilization. In particular, implementation of must-access PDMPs led to a 12 percent decrease in the MME amount of opioids prescribed. This policy also decreased the number of opioid prescriptions, while there was little change in whether workers received opioids. Furthermore, we find that must-access PDMPs contributed to a 12 percent decrease in the likelihood that workers receive opioids on a longer-term basis, among those with opioids. This response was consistent with the policy's goals—reducing the amount of opioids prescribed, including longer-term prescribing. We find that limits on initial opioid prescriptions reduced opioid use among claims with opioids. These limits resulted in a 19 percent decrease in the MME amount of opioids among claims with opioids.

We find some evidence that workers increased the use of other types of care when policies restricted access to opioid prescriptions. For most groups of injuries, we see a decrease in the amount of opioids prescribed due to must-access PDMPs, but relatively small changes in use of other services that may be a substitute for opioids for managing pain. We find substitution towards non-opioid pain prescriptions and interventional pain management services for lacerations and contusions, although for these types of injuries opioid use is not that frequent and we did not find much evidence of declines. More interesting is the evidence for neurologic spine pain cases, where we find evidence consistent with partial substitution towards non-opioid pain medication prescriptions and interventional pain management services. This evidence is potentially significant because, among the injury groups we consider, neurologic spine pain cases have the highest incidence of opioid prescriptions and the highest MME.

Finally, we do not find evidence that either must-access PDMPs or limits on initial prescribing resulted in changes in the duration of temporary disability benefits captured at 12 months of maturity, 
although the estimates are not very precise and hence do not rule out reductions that could be meaningful.

What do these findings imply about the relative effectiveness of must-access PDMPs or limits on initial prescribing? First, the main evidence we find is impacts on amounts of opioids prescribed and numbers of prescriptions, including longer-term prescribing, whereas responses on other dimensions are less evident. Second, in general there is stronger and more robust evidence on the effectiveness of PDMPs. Part of this is because PDMPs act on the number of prescriptions, whereas prescribing limits only have an effect conditional on prescriptions, and even then tend to only reduce MME but not the number of prescriptions. In addition, we have noted that it is a bit more difficult to draw strong statistical conclusions about the effects of initial prescribing limits, in part perhaps because the timing of this policy was relative late, making it more likely that other changes in opioid prescribing and use may have been occurring because of increased focus on the opioid pandemic; empirically, this was manifested in potentially problematic pre-trends in opioid prescribing behavior associated with when and where initial prescribing limits were adopted. As a consequence, we believe our strongest conclusions pertain to the effectiveness of must-access PDMPs.

Our study indicates that opioid-related policies are best understood when we look at multiple dimensions of medical care (and the duration of postinjury recovery). A policy-driven decrease in opioid utilization without a corresponding increase in possible substitutes suggests that some of the initial opioid prescriptions may not have been necessary, that other treatment may not be effective in managing pain, or that workers had problems getting access to other types of care. A limited change in duration of temporary disability benefits adds support for the former interpretation—while workers received fewer opioids, they did not spend more time recovering from their injuries. Unfortunately, we do not have detailed medical information to decide whether the opioids were needed, or to measure how well pain was managed with and without opioid-related policies. Further research efforts are needed to better understand the implications of different types of pain management approaches for workers’ recovery. 


\section{References}

Agarwal, Sunil, John D. Bryan, Hsou Mei Hu, et al. 2019. “Association of State Opioid Duration Limits with Postoperative Opioid Prescribing.” JAMA Network Open 2, no. 12 (December): e1918361.

Alpert, Abby, Sarah E. Dykstra, and Mireille Jacobson. 2020. "How Do Prescription Drug Monitoring Programs Reduce Opioid Prescribing? The Role of Hassle Costs Versus Information.” Working Paper 27584, National Bureau of Economic Research.

American College of Occupational and Environmental Medicine (ACOEM). 2017. Chronic pain guideline.

Bao, Yuhua, Katherine Wen, Phyllis Johnson, et al. 2018. “Assessing the Impact of State Policies for Prescription Drug Monitoring Programs on High-Risk Opioid Prescriptions.” Health Affairs 37, no. 10 (October): 1,596-604.

Ben-Shalom, Yonatan, Megan McIntyre, Jia Pu, et al. 2020. Workers' Compensation and the Opioid Epidemic: State of the Field in Opioid Prescription Management. Mathematica.

Bigos, Stanley J., O. Richard Bowyer, G. Richard Braen, et al. 1994. Acute Low Back Problems in Adults. Clinical Practice Guideline No. 14. AHCPR publication no. 95-0642. Rockville, MD: Agency for Healthcare Policy and Research, Public Health Service, U.S. Department of Health and Human Services.

Buchmueller, Thomas C., and Colleen Carey. 2018. “The Effect of Prescription Drug Monitoring Programs on Opioid Utilization in Medicare.” American Economic Journal: Economic Policy 10, no. 1 (February): 77-112.

Buchmueller, Thomas, Colleen Carey, and Giacomo Meille. 2020. "How Well Do Doctors Know Their Patients? Evidence from a Mandatory Access Prescription Drug Monitoring Program.” Health Economics 29, no. 9 (September): 957-74.

Callaway, Brantly, and Pedro H.C. Sant’Anna. 2021. "Difference-in-differences with Multiple Time Periods.” Journal of Econometrics 225, no. 2 (December): 200-30.

Centers for Disease Control and Prevention (CDC), United States Department of Health and Human Services. 2016. CDC guideline for prescribing opioids for chronic pain - United States, 2016. Morbidity and Mortality Weekly Report 65: 1-49.

Chou, Roger, Amir Qaseem, Vincenza Snow, et al. 2007. "Diagnosis and Treatment of Low Back Pain: A Joint Clinical Practice Guideline from the American College of Physicians and the American Pain Society.” Annals of Internal Medicine 147, no. 7 (October): 478-491.

David, Rena, and Robby Bullis. 2019. Independent Medical Review Decisions January 2014 through December 2018. Oakland, CA: California Workers’ Compensation Institute.

Davis, Corey S., and Derek Carr. 2016. "Physician Continuing Education to Reduce Opioid Misuse, Abuse, and Overdose: Many Opportunities, Few Requirements.” Drug and Alcohol Dependence 163: $100-7$.

Davis, Corey S., Amy Judd Lieberman, Hector Hernandez-Delgado, et al. 2019. “Laws Limiting the Prescribing or Dispensing of Opioids for Acute Pain in the United States: A National Systematic Legal Review.” Drug and Alcohol Dependence 194: 166-72.

Goodman-Bacon, Andrew. 2021. "Difference-in-differences with Variation in Treatment Timing.” Journal of Econometrics 225, no. 2 (December): 254-77.

Grecu, Anca M., Dhaval M. Dave, and Henry Saffer. 2019. "Mandatory Access Prescription Drug Monitoring Programs and Prescription Drug Abuse.” Journal of Policy Analysis and Management 38, no. 1: 181-209.

Haegerich, Tamara M., Leonard J. Paulozzi, Brian J. Manns, and Christopher M. Jones. 2014. "What We Know, and Don't Know, about State and System Level Policy Strategies to Prevent Prescription Drug Overdose.” Drug and Alcohol Dependence 145: 34-47. 
Hayes, Steve, and Alex Swedlow. 2019. California Workers’ Comp Pharmaceutical Utilization \& Reimbursement Part 2: Emerging Outcomes under the MTUS Formulary. Oakland, CA: California Workers' Compensation Institute.

Hegmann, Kurt T., Michael S. Weiss, Kirk Bowden, et al. 2014. “ACOEM Practice Guidelines: Opioids for Treatment of Acute, Subacute, Chronic, and Postoperative Pain. Journal of Occupational and Environmental Medicine. 56, no. 12 (December): e143-59.

Hincapie-Castillo, Juan M., Arnie Goodin, Marie-Christin Possinger, et al. 2020. "Changes in Opioid Use after Florida’s Restriction Law for Acute Pain Prescriptions.” JAMA Network Open 3, no. 2 (February): e200234. doi:10.1001/jamanetworkopen.2020.0234

Horwitz, Jill, Corey S. Davis, Lynn S. McClelland, et al. "The Problem of Data Quality in Analyses of Opioid Regulation: The Case of Prescription Drug Monitoring Programs.” Working Paper 24947. Cambridge, MA: National Bureau of Economic Research.

Jakiela, Pamela. 2021. “Simple Diagnostics for Two-Way Fixed Effects.” Unpublished paper, Williams College.

Kaestner, Robert, and Engy Ziedan. 2019. "Mortality and Socioeconomic Consequences of Prescription Opioids: Evidence from State Policies.” Working Paper 26135. Cambridge, MA: National Bureau of Economic Research.

Maclean, Johanna Catherine, Justine Mallatt, Christopher J. Ruhm, and Kosali Simon. 2020. "Economic Studies on the Opioid Crisis: A Review.” NBER Working Paper No. 28067. Cambridge, MA: National Bureau of Economic Research.

Mallatt, Justine. 2022. "Policy-induced Substitution to Illicit Drugs and Implications for Law Enforcement Activity.” American Journal of Health Economics 8, no. 1 (winter): 30-64.

Mallatt, Justine. 2018. “The Effect of Prescription Drug Monitoring Programs on Opioid Prescriptions and Heroin Crime Rates.” Available at SSRN 3050692.

Meer, Jonathan, and Jeremy West. 2016. "Effects of the Minimum Wage on Employment Dynamics." Journal of Human Resources 56, no. 2 (Spring): 500-22.

Meinhofer, Angélica. 2018. "Prescription Drug Monitoring Programs: The Role of Asymmetric Information on Drug Availability and Abuse.” American Journal of Health Economics 4, no. 4 (Fall)): 504-26.

Pasquale, Margaret K., Richard L. Sheer, Jack Mardekian, et al. 2017. "Educational Intervention for Physicians to Address the Risk of Opioid Abuse.” Journal of Opioid Management 13, no. 5 (September/October): 303-13.

The Pew Charitable Trusts. 2016. Prescription Drug Monitoring Programs: Evidence-based Practices to Optimize Prescriber Use. Philadelphia, PA.

Rothkin, Karen. 2018. Workers' Compensation Prescription Drug Regulations: A National Inventory, 2018. Cambridge, MA: Workers Compensation Research Institute.

Sacks, Daniel W., Alex Hollingsworth, Thuy D. Nguyen, and Kosali Simon. 2019. "Can Policy Affect Initiation of Addictive Substance Use? Evidence from Opioid Prescribing.” Working Paper 25974. Cambridge, MA: National Bureau of Economic Research.

Savych, Bogdan, and Olesya Fomenko. 2019. Workers' Compensation Medical Prices and Outcomes of Injured Workers. Cambridge, MA: Workers Compensation Research Institute.

Savych, Bogdan, David Neumark, and Randall Lea. 2019. "Do Opioids Help Injured Workers Recover and Get Back to Work? The Impact of Opioid Prescriptions on Duration of Temporary Disability.” Industrial Relations 58, no. 4 (October): 549-90.

Shah, Anuj, Corey J. Hayes, and Bradley C. Martin. 2017. "Characteristics of Initial Prescription Episodes and Likelihood of Long-Term Opioid Use-United States, 2006-2015.” Morbidity and Mortality Weekly Report 66, no. 10 (March): 265-69.

Sun, Liyang, and Sarah Abraham. 2021. "Estimating Dynamic Treatment Effects in Event Studies with 
Heterogeneous Treatment Effects.” Journal of Econometrics 225, no. 2 (December)): 175-99.

Texas Department of Insurance (TDI), Workers' Compensation Research and Evaluation Group. 2013. Impact of the Texas Pharmacy Closed Formulary: A Preliminary Report Based on 12-month Injuries with 9-month Services.

Texas Department of Insurance (TDI), Workers’ Compensation Research and Evaluation Group. 2019. Analysis of Injured Employee Outcomes after the Texas Pharmacy Closed Formulary.

Thumula, Vennela. 2014. Impact of Physician Dispensing of Opioid Use. Cambridge, MA: Workers Compensation Research Institute.

Thumula, Vennela. 2017. Impact of Kentucky Opioid Reforms. Cambridge, MA: Workers Compensation Research Institute.

Thumula, Vennela, Dongchun Wang, and Te-Chun Liu. 2019. Interstate Variations in Dispensing of Opioids, 5th Edition. Cambridge, MA: Workers Compensation Research Institute.

Victor, Richard, Olesya Fomenko, and Jonathan Gruber. 2015. Will the Affordable Care Act Shift Claims to Workers' Compensation Payors? Cambridge, MA: Workers Compensation Research Institute.

Wang, Dongchun. 2017. Longer-term Dispensing of Opioids, 4th Edition. Cambridge, MA: Workers Compensation Research Institute.

Wang, Lucy Xiaolu. 2021. "The Complementarity of Drug Monitoring Programs and Health IT for Reducing Opioid-related Mortality and Morbidity.” Health Economics 30, no. 9 (September): 2,02646.

Wen, Hefei, Jason M. Hockenberry, Philip J. Jeng, and Yuhua Bao. 2019. "Prescription Drug Monitoring Program Mandates: Impact on Opioid Prescribing and Related Hospital Use. Health Affairs 38, no. 9 (September): 1,550-56.

Weiss, Elaine, Griffin Murphy, and Leslie I. Boden. 2019. Workers' Compensation: Benefits, Costs and Coverage (2017 Data). Washington, D.C.: National Academy of Social Insurance.

Wooldridge, Jeffrey M. 2021. “Two-Way Fixed Effects, the Two-Way Mundlak Regression, and Difference-in-Differences Estimators.” Unpublished paper, Michigan State University.

Yee, Christine., Steve Pizer, and O., Fomenko. 2015. Why Surgery Rates Vary? Cambridge, MA: Workers Compensation Research Institute.

Ziedan, Engy, and Robert Kaestner. 2020. "Effect of Prescription Opioids and Prescription Opioid Control Policies on Infant Health.” Working Paper 26749. Cambridge, MA: National Bureau of Economic Research. 
Figure 1 Time Series of MME and Percentage of Claims with Opioids

\section{A. MME per Claim}

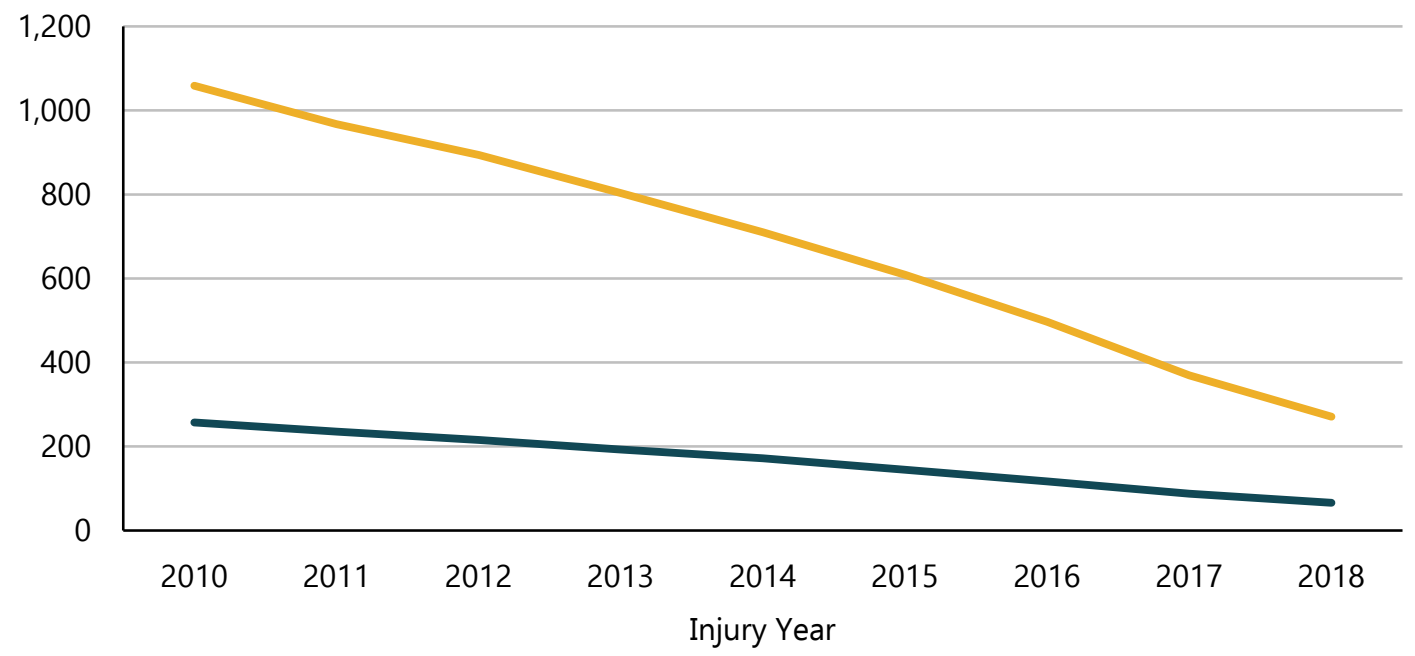

All Claims Claims with More Than 7 Days of Lost Work Time

\section{B. Percentage of Claims with Opioids}

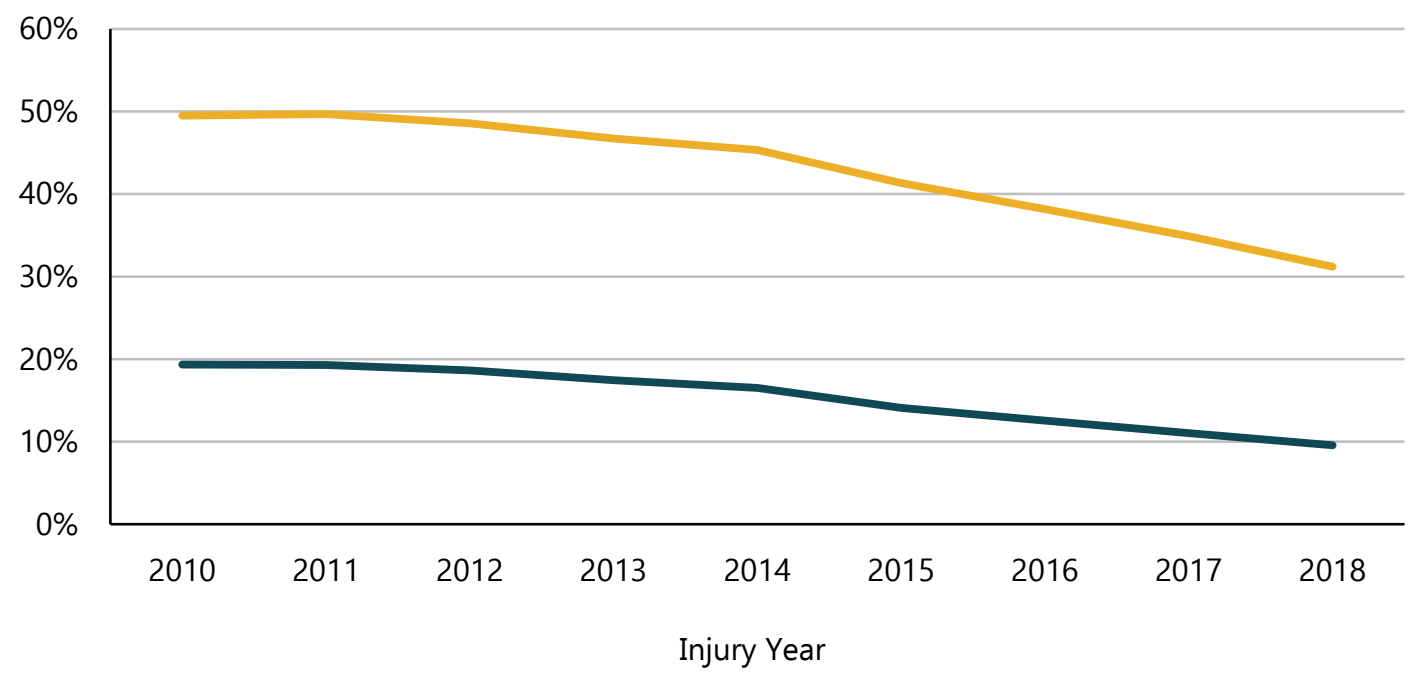

All Claims Claims with More Than 7 Days of Lost Work Time

Notes: Average for claims at 12 months of maturity. Injury year 2010 indicates injuries that occurred between October 1, 2009, and September 30, 2010. Other years denoted similarly. 2018 includes injuries through March 31, 2018, evaluated through March 31, 2019.

Key: MME: morphine milligram equivalent amount of opioids. 


\section{A. PDMPs}

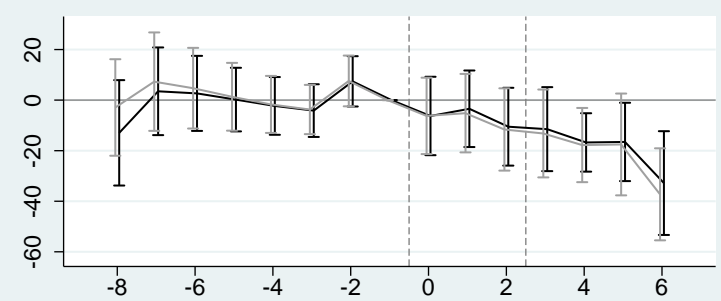

\section{B. Limits on Opioid Prescriptions}

MME for all claims

Any opioids for all claims
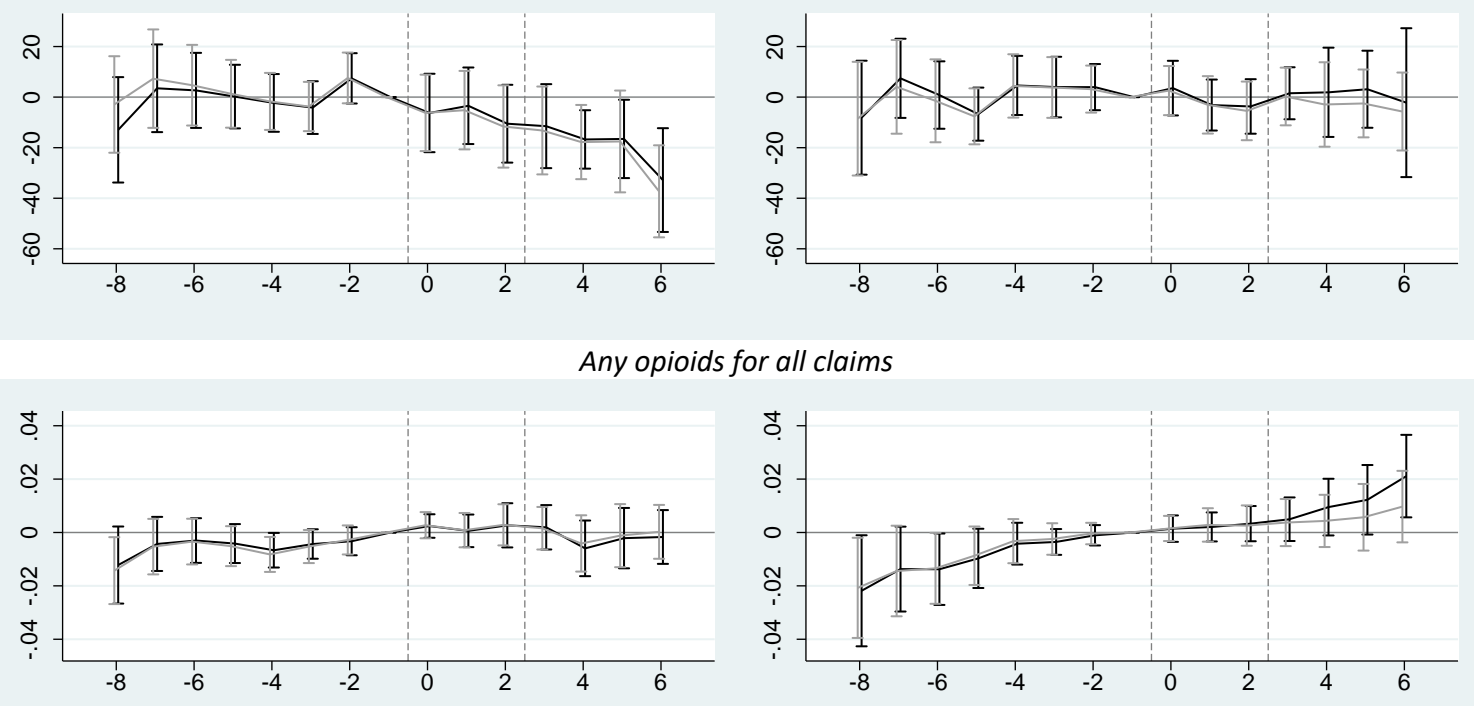

Number of opioid prescriptions for all claims
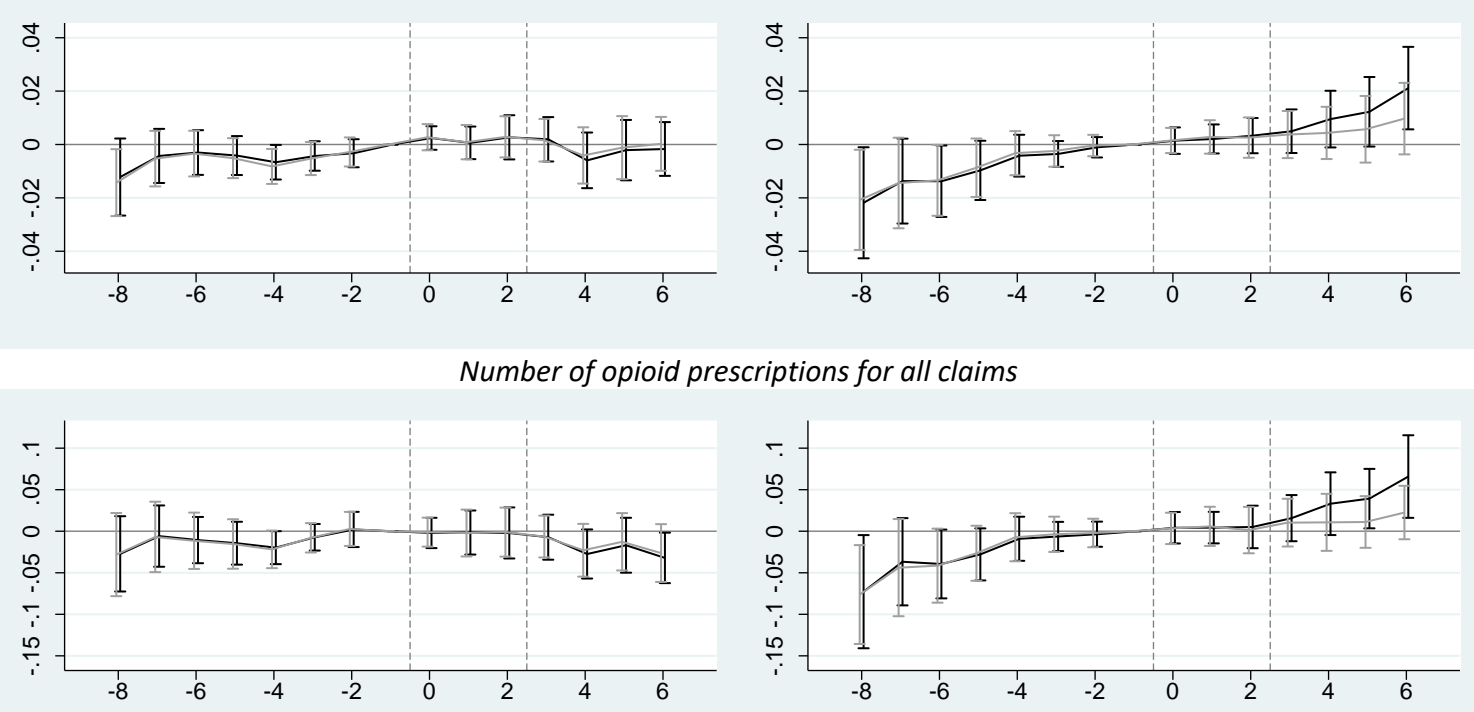

MME for claims with opioids
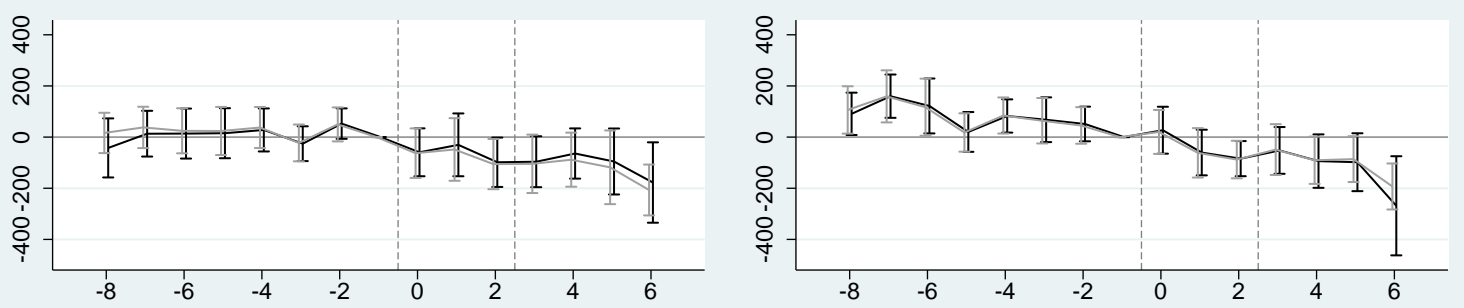

Number of opioid prescriptions, claims with opioids
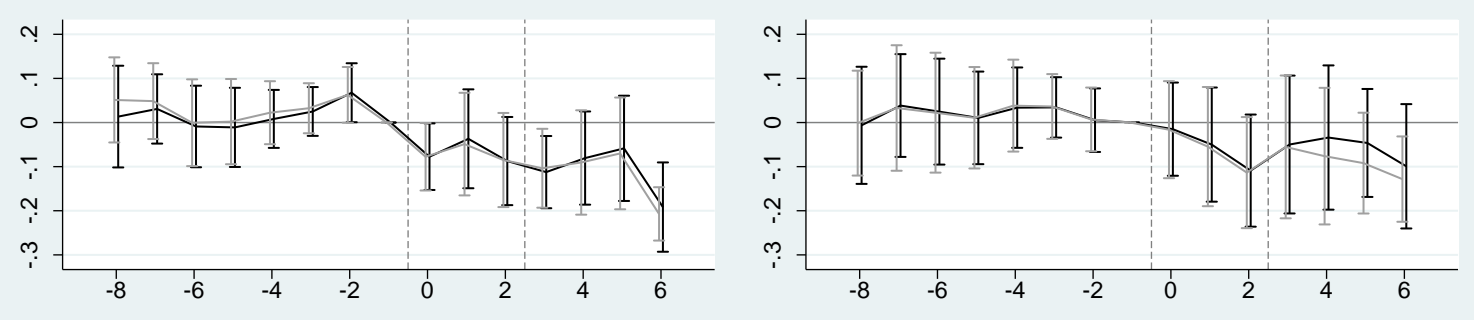

TWFE

Sun and Abraham

Notes: Coefficient estimates from the event-study analysis comparing differences between treated and control states by quarter before and after the date of policy implementation (displayed on horizontal axes) for claims at 12 months of maturity. The two full vertical lines indicate the beginning of the partial policy impact (quarter 0 ) and then the period when full impact begins (quarter 3 ). Controls are included for industry composition, average county-level unemployment rate, average county-level median household income, average county-level percentage disabled, and average county-level percentage without health insurance. We also control for state and (unique) quarter fixed effects. Ninety-five percent confidence intervals are shown as the vertical lines through the plotted points. Quarter "-8" reflects difference for all quarters up to and including quarter -8 . Quarter " 6 " reflects difference for all quarters 6 or greater.

Key: MME: morphine milligram equivalent amount of opioids; PDMPs: must-access prescription drug monitoring programs. 
Figure 3 Event-Study Regressions for Limits on Opioid Prescriptions Excluding Late Adopters (IN, KY, LA, MD, MN, NV, NC) - Parallels Panel B of Figure 2

MME for all claims

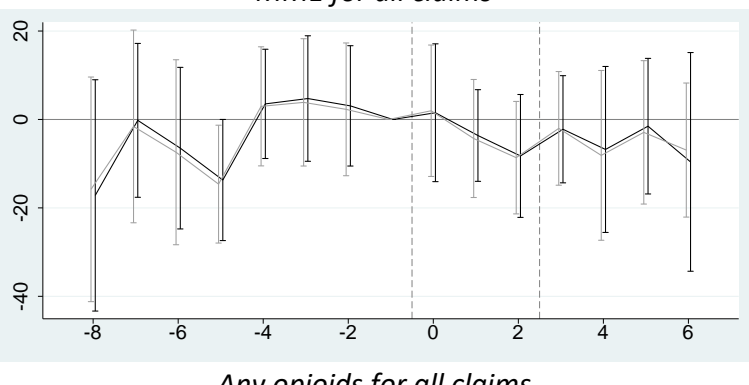

Any opioids for all claims

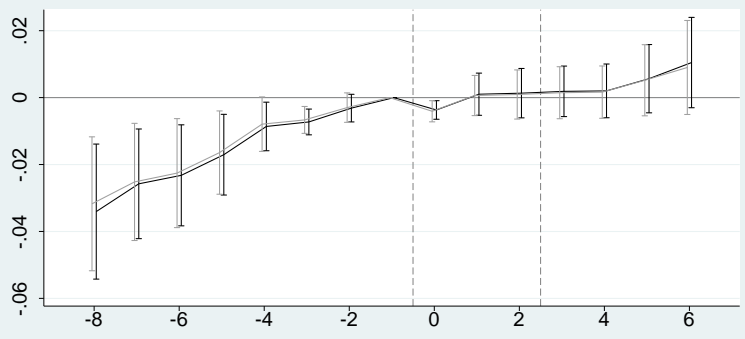

Number of opioid prescriptions for all claims

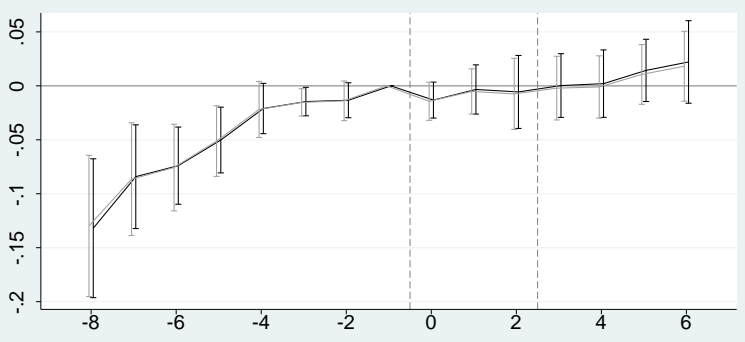

MME for claims with opioids

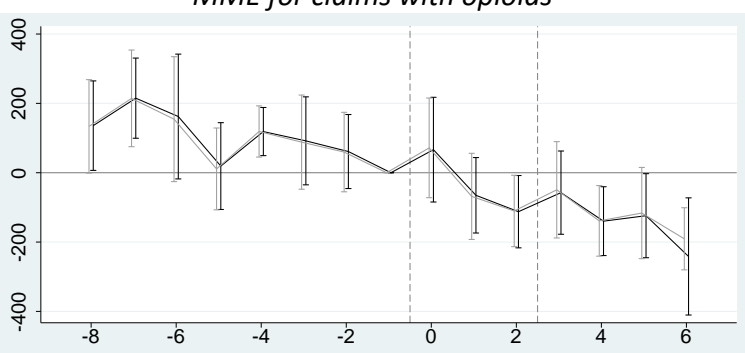

Number of opioid prescriptions, claims with opioids

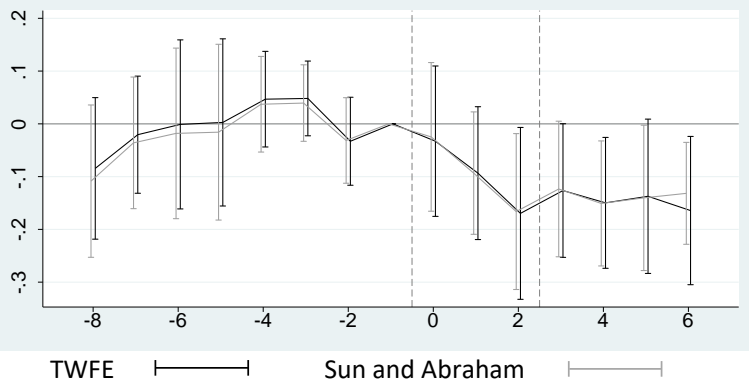

Notes: Coefficient estimates from the event-study analysis comparing differences between treated and control states by quarter before and after the date of policy implementation (displayed on horizontal axes) for claims at 12 months of maturity. The two full vertical lines indicate the beginning of the partial policy impact (quarter 0 ) and then the period when full impact begins (quarter 3). Controls are included for industry composition, average county-level unemployment rate, average county-level median household income, average county-level percentage disabled, and average county-level percentage without health insurance. We also control for state and (unique) quarter fixed effects. Ninety-five percent confidence intervals are shown as the vertical lines through the plotted points. This figure differs from Figures 2 by excluding the states that adopted initial opioid prescribing limits late (see Table 1). Quarter "-8" reflects difference for all quarters up to and including quarter -8 . Quarter " 6 " reflects difference for all quarters 6 or greater.

Key: MME: morphine milligram equivalent amount of opioids; PDMPs: must-access prescription drug monitoring programs. Grey lines represent estimates from Sun and Abraham (2021). 
Table 1 Effective Dates of Must-Access PDMPs and Limits on Initial Opioid Prescriptions

\begin{tabular}{|c|c|c|c|}
\hline \multirow[b]{2}{*}{ State } & \multirow{2}{*}{$\begin{array}{c}\text { Effective Date of } \\
\text { Must-Access PDMP }\end{array}$} & \multicolumn{2}{|c|}{ Limits on Initial Opioid Prescriptions } \\
\hline & & Effective Date & Days of Supply Limits \\
\hline \multicolumn{4}{|l|}{ Alabama } \\
\hline Arkansas & Aug-17 & Aug-18 & 7 \\
\hline Arizona & Oct-17 & Apr-18 & $5^{a}$ \\
\hline California & Oct-18 & & \\
\hline Connecticut & Oct-15 & Jul-16 & 7 \\
\hline Delaware & Mar-12 & Apr-17 & 7 \\
\hline Florida & Jul-18 & Jul-18 & 3 or $7^{b}$ \\
\hline Georgia & Jul-18 & & \\
\hline \multicolumn{4}{|l|}{ Hawaii } \\
\hline Illinois & Jan-18 & $--^{c}$ & \\
\hline Indiana & Jan-19 & Jul-17 & 7 \\
\hline lowa & Jul-18 & & \\
\hline \multicolumn{4}{|l|}{ Kansas } \\
\hline Kentucky & Jul-12 & Jun-17 & 3 \\
\hline Louisiana & Aug-14 & Aug-17 & 7 \\
\hline Maryland & Jul-18 & May-17 & $--^{d}$ \\
\hline Massachusetts & Jan-16 & Mar-16 & 7 \\
\hline Michigan & Jun-18 & Jul-18 & 7 \\
\hline Minnesota & & Jul-17 & $4^{e}$ \\
\hline \multicolumn{4}{|l|}{ Mississippi } \\
\hline Missouri & & Aug- $18^{c}$ & 7 \\
\hline Nevada & Oct-15 & Jun-17 & $14^{f}$ \\
\hline New Jersey & Nov-15 & May-17 & $5^{g}$ \\
\hline New Mexico & Sep-12 & & \\
\hline New York & Aug-13 & Jul-16 & 7 \\
\hline North Carolina & Nov-18 & Jan-18 & 5 \\
\hline Oklahoma & Nov-15 & & \\
\hline Pennsylvania & Jul-15 & Jan-17 & $7^{i}$ \\
\hline South Carolina & May-17 & May-18 & 7 \\
\hline Tennessee & Apr-13 & Jul-18 & $--^{j}$ \\
\hline Texas & Sep-19 & & \\
\hline Virginia & Jul-15 & Mar-17 & $7^{k}$ \\
\hline Wisconsin & Apr-17 & Apr-18 & $3-5$ \\
\hline
\end{tabular}

Note: We used multiple sources of information for this table, including Thumula et al. (2019), Davis et al. (2019), and online reports and articles on timing of policy adoption.

${ }^{a}$ In Arizona, the limit on an initial prescription for a Schedule II controlled substance that is an opioid following a surgical procedure is no more than a 14-day supply.

${ }^{\mathrm{b}}$ Florida limits initial opioid prescriptions to 3 days of supply, or 7 days if the provider determines the lack of available alternative treatment.

${ }^{\mathrm{c}}$ Illinois and Missouri had limits of 30 days of supply in earlier years, which were not part of the legislative trend toward limiting initial opioid prescriptions.

${ }^{d}$ In Maryland, the law requires prescribing opioids with the lowest effective dose, without limiting the number of days of supply.

e Minnesota has a four-day limit only for dental and refractive surgery pain.

${ }^{f}$ Nevada limits opioids to 14 days of supply and 90 morphine milligram equivalent amount of opioids (MME) per day.

${ }^{\mathrm{g}} \mathrm{New}$ Jersey limits prescriptions to the lowest effective dose.

"The "targeted controlled substances" are Schedule II and III opioids and narcotics per the North Carolina Controlled Substances Act, specifically those listed in N.C. Gen. Stat. §90-90(1), (2) or 90-91(d). This provision does not apply to opioid prescriptions administered in a hospital, nursing home, hospice facility, or residential care facility.

' Pennsylvania's 7-day supply limit is for emergency department visits, urgent care, and hospital observation patient only.

${ }^{j}$ Tennessee has a cascading limit for initial opioid prescriptions. For pain, the limit is 10 days of supply with the maximum amount of $500 \mathrm{MME}$. For post-surgical pain, the limit is 20 days of supply with a maximum amount of $850 \mathrm{MME}$. If there is well-justified medical need, the limit is 30 days of supply, with a maximum total of 1,200 MME.

${ }^{k}$ Virginia limits initial opioids to 7 days of supply, or 14 days for post-surgical pain. 
Table 3 Estimated Effect of Policies on Opioid Utilization by Injury Group

\begin{tabular}{|c|c|c|c|c|c|c|c|c|}
\hline & (1) & (2) & (3) & (4) & (5) & (6) & (7) & (8) \\
\hline & Fractures & $\begin{array}{c}\text { Lacerations } \\
\text { and } \\
\text { Contusions }\end{array}$ & Inflammations & $\begin{array}{l}\text { Neurologic } \\
\text { Spine Pain }\end{array}$ & $\begin{array}{c}\text { Spine Sprains } \\
\text { and Strains }\end{array}$ & $\begin{array}{c}\text { Other Sprains } \\
\text { and Strains }\end{array}$ & $\begin{array}{c}\text { Upper } \\
\text { Extremity } \\
\text { Neurologic }\end{array}$ & Other Injuries \\
\hline \multicolumn{9}{|l|}{ A. MME for all claims } \\
\hline \multirow[t]{2}{*}{ Must-access PDMP } & 2.1268 & 3.4728 & -46.3165 & $-160.4689 * *$ & $-33.6107 * *$ & $-13.3391^{*}$ & 3.1276 & -6.6082 \\
\hline & $(17.9836)$ & $(3.0668)$ & $(28.2368)$ & (66.0131) & $(14.6385)$ & $(7.8328)$ & (35.0599) & $(7.9022)$ \\
\hline Observations & 1,118 & 1,118 & 1,118 & 1,118 & 1,118 & 1,118 & 1,118 & 1,118 \\
\hline Mean of outcome variable & 354.586 & 34.341 & 360.143 & 1232.287 & 162.615 & 139.149 & 242.066 & 123.937 \\
\hline$\%$ effect for PDMPs & $1 \%$ & $10 \%$ & $-13 \%$ & $-13 \% * *$ & $-21 \% * *$ & $-10 \% *$ & $1 \%$ & $-5 \%$ \\
\hline \multicolumn{9}{|l|}{ B. Any opioids for all claims } \\
\hline \multirow[t]{2}{*}{ Must-access PDMP } & 0.0109 & $0.0129 *$ & 0.0094 & 0.0116 & 0.0154 & 0.0107 & -0.0058 & 0.0063 \\
\hline & (0.0109) & $(0.0066)$ & $(0.0105)$ & $(0.0131)$ & $(0.0120)$ & $(0.0088)$ & $(0.0225)$ & $(0.0044)$ \\
\hline Observations & 1,118 & 1,118 & 1,118 & 1,118 & 1,118 & 1,118 & 1,118 & 1,118 \\
\hline Mean of outcome variable & 0.330 & 0.083 & 0.285 & 0.450 & 0.185 & 0.166 & 0.327 & 0.127 \\
\hline$\%$ effect for PDMPs & $3 \%$ & $15 \%$ & $3 \%$ & $3 \%$ & $8 \%$ & $6 \%$ & $-2 \%$ & $5 \%$ \\
\hline \multicolumn{9}{|c|}{ C. Number of opioid prescriptions among all claims } \\
\hline \multirow[t]{2}{*}{ Must-access PDMP } & 0.0488 & $0.0226^{*}$ & -0.0032 & 0.1020 & 0.0090 & 0.0029 & 0.0663 & 0.0135 \\
\hline & $(0.0448)$ & $(0.0116)$ & $(0.0581)$ & $(0.1181)$ & $(0.0334)$ & $(0.0240)$ & $(0.0987)$ & $(0.0177)$ \\
\hline Observations & 1,118 & 1,118 & 1,118 & 1,118 & 1,118 & 1,118 & 1,118 & 1,118 \\
\hline Mean of outcome variable & 0.972 & 0.142 & 1.015 & 2.332 & 0.472 & 0.451 & 0.837 & 0.336 \\
\hline$\%$ effect for PDMPs & $5 \%$ & $16 \% *$ & $0 \%$ & $4 \%$ & $2 \%$ & $1 \%$ & $8 \%$ & $4 \%$ \\
\hline \multicolumn{9}{|c|}{ D. MME for claims with opioids } \\
\hline \multirow[t]{2}{*}{ Must-access PDMP } & -11.9202 & 4.0842 & $-208.2098 * * *$ & $-368.4191^{* *}$ & $-188.6995^{* *}$ & $-93.2086^{* *}$ & -51.6436 & $-98.4392^{* *}$ \\
\hline & $(40.1921)$ & $(28.3852)$ & $(62.6083)$ & (140.9399) & $(81.8014)$ & $(42.7971)$ & (78.5488) & $(44.2494)$ \\
\hline \multirow[t]{2}{*}{ Limits on initial opioid Rx } & $-284.0476 * * *$ & -26.5378 & $-146.7429 *$ & $-418.3622 * * *$ & 1.9073 & $-140.9779 * * *$ & -21.4663 & $-158.9752 * * *$ \\
\hline & $(67.5311)$ & $(40.4862)$ & (79.5444) & $(132.5973)$ & (95.7819) & $(39.0382)$ & (74.5350) & $(42.2049)$ \\
\hline Observations & 1,118 & 1,118 & 1,118 & 1,118 & 1,118 & 1,118 & 1,095 & 1,118 \\
\hline Mean of outcome variable & 1094.129 & 432.471 & 1289.048 & 2768.341 & 909.103 & 865.373 & 750.724 & 1001.242 \\
\hline$\%$ effect for PDMPs & $-1 \%$ & $1 \%$ & $-16 \% * * *$ & $-13 \% * *$ & $-21 \% * *$ & $-11 \% * *$ & $-7 \%$ & $-10 \% * *$ \\
\hline$\%$ effect for initial limits & $-26 \% * * *$ & $-6 \%$ & $-11 \% *$ & $-15 \% * * *$ & $0 \%$ & $-16 \% * * *$ & $-3 \%$ & $-16 \% * * *$ \\
\hline \multicolumn{9}{|c|}{ E. Number of opioid prescriptions among claims with opioids } \\
\hline \multirow[t]{2}{*}{ Must-access PDMP } & -0.0542 & -0.0151 & $-0.2612^{* * *}$ & -0.1716 & $-0.1995 * * *$ & $-0.1618^{* * *}$ & 0.0227 & $-0.1345^{* *}$ \\
\hline & $(0.0510)$ & $(0.0280)$ & $(0.0732)$ & $(0.1124)$ & $(0.0635)$ & $(0.0440)$ & $(0.1120)$ & $(0.0516)$ \\
\hline \multirow[t]{2}{*}{ Limits on initial opioid $\mathrm{Rx}$} & -0.0966 & $0.0913 * * *$ & 0.0588 & 0.1939 & 0.1115 & $-0.1157^{* *}$ & 0.1218 & -0.0281 \\
\hline & $(0.0691)$ & $(0.0264)$ & $(0.0825)$ & $(0.1768)$ & $(0.0857)$ & $(0.0520)$ & $(0.1389)$ & $(0.0719)$ \\
\hline Observations & 1,118 & 1,118 & 1,118 & 1,118 & 1,118 & 1,118 & 1,095 & 1,118 \\
\hline Mean of outcome variable & 2.942 & 1.702 & 3.566 & 5.189 & 2.547 & 2.716 & 2.557 & 2.651 \\
\hline$\%$ effect for PDMPs & $-2 \%$ & $-1 \%$ & $-7 \% * * *$ & $-3 \%$ & $-8 \% * * *$ & $-6 \% * * *$ & $1 \%$ & $-5 \% * *$ \\
\hline$\%$ effect for initial limits & $-3 \%$ & $5 \% * * *$ & $2 \%$ & $4 \%$ & $4 \%$ & $-4 \% * *$ & $5 \%$ & $-1 \%$ \\
\hline
\end{tabular}

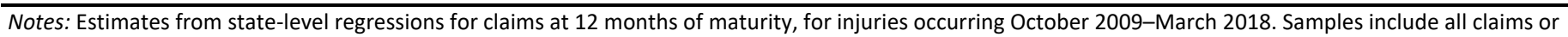
claims with opioids (as indicated) aggregated to quarter of injury and state. Each observation is weighted by the number of claims represented. Controls are included for industry composition, average county-level unemployment rate, average county-level median household income, average county-level percentage disabled, and average county-level percentage without health insurance. We also control for state and quarter fixed effects.

$*, * *, * * *$ Statistically significant at the 10 percent, 5 percent, and 1 percent level, respectively. (The asterisks reported for the "\% effect" rows are the same as for the corresponding coefficient estimates.) Standard errors are clustered by state. To account for partial exposure for injuries for which the policy changed during the 12 months the data capture, we define the variable denoting the policy change as 1 if it was in effect for all 12 months of the postinjury exposure,

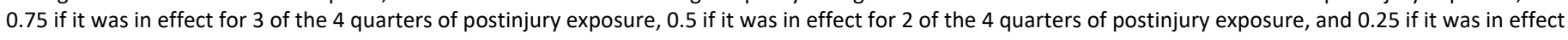
for only the last quarter of the 4 quarters of postinjury exposure.

Key: MME: morphine milligram equivalent amount of opioids; PDMP: must-access prescription drug monitoring program; Rx: prescription. 
Table 4 Effect of Policies on Problematic Opioid Use Indicators

\begin{tabular}{|c|c|c|c|c|c|c|}
\hline & (1) & (2) & (3) & (4) & (5) & (6) \\
\hline Outcome & $\begin{array}{c}\text { Longer- } \\
\text { Term } \\
\text { Opioid } \\
\text { Prescribing }\end{array}$ & $\begin{array}{c}\text { More Than } \\
90 \text { Days of } \\
\text { Opioids } \\
\text { Prescribed }\end{array}$ & $\begin{array}{l}\text { Daily Dose } \\
\text { Greater Than } \\
120 \mathrm{mg}\end{array}$ & $\begin{array}{l}\text { Longer-Term } \\
\text { Opioid } \\
\text { Prescribing }\end{array}$ & $\begin{array}{l}\text { More Than } \\
90 \text { Days of } \\
\text { Opioids } \\
\text { Prescribed }\end{array}$ & $\begin{array}{c}\text { Daily Dose } \\
\text { Greater Than } \\
120 \mathrm{mg}\end{array}$ \\
\hline Sample & All Claims & All Claims & All Claims & $\begin{array}{c}\text { Claims with } \\
\text { Opioids }\end{array}$ & $\begin{array}{c}\text { Claims with } \\
\text { Opioids }\end{array}$ & $\begin{array}{l}\text { Claims with } \\
\text { Opioids }\end{array}$ \\
\hline \multirow[t]{2}{*}{ Must-access PDMP } & -0.0001 & -0.0002 & -0.0002 & $-0.0070^{* * *}$ & -0.0046 & -0.0006 \\
\hline & $(0.0007)$ & $(0.0009)$ & $(0.0002)$ & $(0.0021)$ & $(0.0046)$ & $(0.0012)$ \\
\hline \multirow[t]{2}{*}{ Limits on initial opioid Rx } & & & & -0.0009 & 0.0005 & -0.0005 \\
\hline & & & & $(0.0047)$ & $(0.0076)$ & $(0.0012)$ \\
\hline Observations & 1,118 & 1,118 & 1,118 & 1,118 & 1,118 & 1,118 \\
\hline Mean of outcome variable & 0.0097 & 0.0111 & 0.0017 & 0.0601 & 0.1011 & 0.0159 \\
\hline$\%$ effect for PDMPs & $-1 \%$ & $-2 \%$ & $-12 \%$ & $-12 \% * * *$ & $-5 \%$ & $-4 \%$ \\
\hline$\%$ effect for initial limits & & & & $-1 \%$ & $0 \%$ & $-3 \%$ \\
\hline \multicolumn{7}{|c|}{$\begin{array}{l}\text { Notes: Estimates from state-level regressions for claims at } 12 \text { months of maturity, for injuries occurring October 2009-March } \\
\text { 2018. Samples include claims with opioids aggregated to quarter of injury and state. Each observation is weighted by the number } \\
\text { of claims represented. Longer-term opioid prescriptions are defined as having prescriptions within the first three months after an } \\
\text { injury and three or more filled opioid prescriptions between the 7th and } 12 \text { th months after an injury. Controls are included for } \\
\text { industry composition, average county-level unemployment rate, average county-level median household income, average county- } \\
\text { level percentage disabled, and average county-level percentage without health insurance. We also control for state and quarter } \\
\text { fixed effects. }{ }^{*}, * * * * * \text { Statistically significant at the } 10 \text { percent, } 5 \text { percent, and } 1 \text { percent level, respectively. (The asterisks } \\
\text { reported for the "\% effect" rows are the same as for the corresponding coefficient estimates.) Standard errors are clustered by } \\
\text { state. Data are aggregated to quarterly frequency. To account for partial exposure for injuries for which the policy changed during } \\
\text { the } 12 \text { months the data capture, we define the variable denoting the policy change as } 1 \text { if it was in effect for all } 12 \text { months of the } \\
\text { postinjury exposure, } 0.75 \text { if it was in effect for } 3 \text { of the } 4 \text { quarters of postinjury exposure, } 0.5 \text { if it was in effect for } 2 \text { of the } 4 \\
\text { quarters of postinjury exposure, and } 0.25 \text { if it was in effect for only the last quarter of the } 4 \text { quarters of postinjury exposure. } \\
\text { Key: PDMP: must-access prescription drug monitoring program; Rx: prescription. }\end{array}$} \\
\hline
\end{tabular}


Table 5 Estimated Effect of Policies on Problematic Opioid Use Indicators by Injury Group

\begin{tabular}{|c|c|c|c|c|c|c|c|c|}
\hline & Fractures & $\begin{array}{c}\text { Lacerations } \\
\text { and } \\
\text { Contusions }\end{array}$ & Inflammations & $\begin{array}{l}\text { Neurologic } \\
\text { Spine Pain }\end{array}$ & $\begin{array}{c}\text { Spine Sprains } \\
\text { and Strains }\end{array}$ & $\begin{array}{c}\text { Other Sprains } \\
\text { and Strains }\end{array}$ & $\begin{array}{c}\text { Upper } \\
\text { Extremity } \\
\text { Neurologic } \\
\end{array}$ & $\begin{array}{c}\begin{array}{c}\text { Other } \\
\text { injuries }\end{array} \\
\end{array}$ \\
\hline \multicolumn{9}{|c|}{ A. Longer-term opioid prescribing among all claims } \\
\hline \multirow[t]{2}{*}{ Must-access PDMP } & 0.0019 & 0.0002 & -0.0005 & 0.0045 & -0.0006 & -0.0002 & 0.0035 & 0.0002 \\
\hline & $(0.0014)$ & $(0.0001)$ & $(0.0024)$ & $(0.0055)$ & $(0.0011)$ & $(0.0006)$ & $(0.0039)$ & $(0.0006)$ \\
\hline Observations & 1,118 & 1,118 & 1,118 & 1,118 & 1,118 & 1,118 & 1,118 & 1,118 \\
\hline Mean of outcome variable & 0.014 & 0.001 & 0.026 & 0.087 & 0.009 & 0.008 & 0.015 & 0.006 \\
\hline$\%$ effect for PDMPs & $13 \%$ & $20 \%$ & $-2 \%$ & $5 \%$ & $-6 \%$ & $-3 \%$ & $24 \%$ & $3 \%$ \\
\hline \multicolumn{9}{|c|}{ B. More than 90 days of opioids prescribed among all claims } \\
\hline \multirow[t]{2}{*}{ Must-access PDMP } & 0.0019 & 0.0001 & -0.0020 & 0.0061 & -0.0011 & 0.0002 & 0.0023 & -0.0000 \\
\hline & $(0.0017)$ & $(0.0002)$ & $(0.0024)$ & $(0.0068)$ & $(0.0015)$ & $(0.0007)$ & $(0.0035)$ & $(0.0007)$ \\
\hline Observations & 1,118 & 1,118 & 1,118 & 1,118 & 1,118 & 1,118 & 1,118 & 1,118 \\
\hline Mean of outcome variable & 0.019 & 0.001 & 0.024 & 0.113 & 0.013 & 0.008 & 0.015 & 0.007 \\
\hline$\%$ effect for PDMPs & $10 \%$ & $8 \%$ & $-8 \%$ & $5 \%$ & $-8 \%$ & $2 \%$ & $16 \%$ & $0 \%$ \\
\hline \multicolumn{9}{|c|}{ C. Daily dose greater than $120 \mathrm{mg}$ among all claims } \\
\hline \multirow{2}{*}{ Must-access PDMP } & -0.0002 & -0.0001 & -0.0004 & $-0.0019 * *$ & -0.0002 & 0.0002 & -0.0011 & -0.0002 \\
\hline & $(0.0005)$ & $(0.0001)$ & $(0.0008)$ & $(0.0009)$ & $(0.0001)$ & $(0.0002)$ & $(0.0014)$ & $(0.0002)$ \\
\hline Observations & 1,118 & 1,118 & 1,118 & 1,118 & 1,118 & 1,118 & 1,118 & 1,118 \\
\hline Mean of outcome variable & 0.005 & 0.001 & 0.005 & 0.006 & 0.001 & 0.002 & 0.005 & 0.002 \\
\hline \% effect for PDMPs & $-4 \%$ & $-20 \%$ & $-8 \%$ & $-34 \% * *$ & $-17 \%$ & $11 \%$ & $-21 \%$ & $-13 \%$ \\
\hline \multicolumn{9}{|c|}{ D. Longer-term opioid prescribing among claims with opioids } \\
\hline \multirow[t]{2}{*}{ Must-access PDMP } & 0.0003 & 0.0001 & $-0.0136 * * *$ & -0.0097 & $-0.0094 * * *$ & $-0.0068^{* *}$ & -0.0010 & $-0.0055^{* *}$ \\
\hline & $(0.0031)$ & $(0.0011)$ & $(0.0040)$ & $(0.0065)$ & $(0.0028)$ & $(0.0029)$ & $(0.0072)$ & $(0.0024)$ \\
\hline \multirow[t]{2}{*}{ Limits on initial opioid $\mathrm{Rx}$} & -0.0013 & $0.0043^{* *}$ & 0.0089 & 0.0064 & 0.0055 & -0.0030 & 0.0122 & -0.0002 \\
\hline & $(0.0042)$ & $(0.0017)$ & $(0.0054)$ & $(0.0084)$ & $(0.0052)$ & $(0.0036)$ & $(0.0097)$ & $(0.0043)$ \\
\hline Observations & 1,118 & 1,118 & 1,118 & 1,118 & 1,118 & 1,118 & 1,095 & 1,118 \\
\hline Mean of outcome variable & 0.043 & 0.012 & 0.092 & 0.195 & 0.051 & 0.047 & 0.045 & 0.049 \\
\hline$\%$ effect for PDMPs & $1 \%$ & $1 \%$ & $-15 \% * * *$ & $-5 \%$ & $-19 \% * * *$ & $-14 \% * *$ & $-2 \%$ & $-11 \%^{* *}$ \\
\hline$\%$ effect for initial limits & $-3 \%$ & $35 \%$ & $10 \%$ & $3 \%$ & $11 \%$ & $-6 \%$ & $27 \%$ & $0 \%$ \\
\hline \multicolumn{9}{|c|}{ E. More than 90 days of opioids prescribed among claims with opioids } \\
\hline \multirow[t]{2}{*}{ Must-access PDMP } & 0.0013 & -0.0007 & $-0.0147 * *$ & 0.0049 & $-0.0130^{*}$ & -0.0010 & 0.0021 & -0.0077 \\
\hline & $(0.0061)$ & $(0.0037)$ & $(0.0066)$ & $(0.0125)$ & $(0.0070)$ & $(0.0040)$ & $(0.0090)$ & $(0.0048)$ \\
\hline \multirow[t]{2}{*}{ Limits on initial opioid $\mathrm{Rx}$} & -0.0015 & 0.0023 & 0.0098 & -0.0015 & $0.0194^{*}$ & -0.0013 & 0.0149 & 0.0047 \\
\hline & $(0.0079)$ & $(0.0041)$ & (0.0089) & $(0.0153)$ & $(0.0107)$ & $(0.0052)$ & $(0.0144)$ & $(0.0061)$ \\
\hline Observations & 1,117 & 1,118 & 1,118 & 1,118 & 1,118 & 1,118 & 1,068 & 1,118 \\
\hline Mean of outcome variable & 0.072 & 0.028 & 0.111 & 0.301 & 0.112 & 0.075 & 0.059 & 0.074 \\
\hline$\%$ effect for PDMPs & $2 \%$ & $-2 \%$ & $-13 \% * *$ & $2 \%$ & $-12 \% *$ & $-1 \%$ & $4 \%$ & $-10 \%$ \\
\hline$\%$ effect for initial limits & $-2 \%$ & $8 \%$ & $9 \%$ & $0 \%$ & $17 \%$ & $-2 \%$ & $25 \%$ & $6 \%$ \\
\hline \multicolumn{9}{|c|}{ F. Daily dose greater than $120 \mathrm{mg}$ among claims with opioids } \\
\hline \multirow[t]{2}{*}{ Must-access PDMP } & -0.0007 & -0.0027 & -0.0009 & $-0.0044^{*}$ & -0.0012 & 0.0029 & -0.0012 & -0.0012 \\
\hline & $(0.0019)$ & $(0.0019)$ & $(0.0031)$ & $(0.0024)$ & $(0.0015)$ & $(0.0019)$ & $(0.0057)$ & $(0.0014)$ \\
\hline \multirow[t]{2}{*}{ Limits on initial opioid $\mathrm{Rx}$} & -0.0011 & -0.0015 & 0.0010 & -0.0012 & 0.0019 & -0.0005 & -0.0059 & 0.0008 \\
\hline & $(0.0027)$ & $(0.0026)$ & $(0.0032)$ & $(0.0027)$ & (0.0019) & $(0.0017)$ & $(0.0051)$ & $(0.0017)$ \\
\hline Observations & 1,117 & 1,118 & 1,118 & 1,118 & 1,118 & 1,118 & 1,068 & 1,118 \\
\hline Mean of outcome variable & 0.019 & 0.012 & 0.023 & 0.015 & 0.011 & 0.017 & 0.021 & 0.017 \\
\hline$\%$ effect for PDMPs & $-4 \%$ & $-23 \%$ & $-4 \%$ & $-29 \% *$ & $-11 \%$ & $18 \%$ & $-6 \%$ & $-7 \%$ \\
\hline$\%$ effect for initial limits & $-6 \%$ & $-13 \%$ & $4 \%$ & $-8 \%$ & $18 \%$ & $-3 \%$ & $-29 \%$ & $5 \%$ \\
\hline \multicolumn{9}{|c|}{$\begin{array}{l}\text { Notes: Estimates from state-level regressions for claims at } 12 \text { months of maturity, for injuries occurring October 2009-March 2018. Samples include } \\
\text { all claims or claims with opioids (as indicated) aggregated to quarter of injury and state. Each observation is weighted by the number of claims } \\
\text { represented. Longer-term opioid prescriptions are defined as having prescriptions within the first three months after an injury and three or more } \\
\text { filled opioid prescriptions between the } 7 \text { th and } 12 \text { th months after an injury. Controls are included for industry composition, average county-level } \\
\text { unemployment rate, average county-level median household income, average county-level percentage disabled, and average county-level } \\
\text { percentage without health insurance. We also control for state and quarter fixed effects. }{ }^{* * *}, * * * \text { Statistically significant at the } 10 \text { percent, } 5 \\
\text { percent, and } 1 \text { percent level, respectively. (The asterisks reported for the "\% effect" rows are the same as for the corresponding coefficient } \\
\text { estimates.) Standard errors are clustered by state. To account for partial exposure for injuries for which the policy changed during the } 12 \text { months } \\
\text { the data capture, we define the variable denoting the policy change as } 1 \text { if it was in effect for all } 12 \text { months of the postinjury exposure, } 0.75 \text { if it was } \\
\text { in effect for } 3 \text { of the } 4 \text { quarters of postinjury exposure, } 0.5 \text { if it was in effect for } 2 \text { of the } 4 \text { quarters of postinjury exposure, and } 0.25 \text { if it was in effect } \\
\text { for only the last quarter of the } 4 \text { quarters of postinjury exposure. } \\
\text { Key: PDMP: must-access prescription drug monitoring program: Rx: prescription. }\end{array}$} \\
\hline
\end{tabular}


Table 6 Estimated Effect of Opioid Policies on Other Care Overall and by Injury Group, All Claims

\begin{tabular}{|c|c|c|c|c|c|c|c|c|}
\hline & (1) & $(2)$ & (3) & (4) & (5) & $(6)$ & (7) & (8) \\
\hline & Fractures & $\begin{array}{c}\text { Lacerations } \\
\text { and } \\
\text { Contusions }\end{array}$ & Inflammations & $\begin{array}{l}\text { Neurologic } \\
\text { Spine Pain }\end{array}$ & $\begin{array}{c}\text { Spine } \\
\text { Sprains and } \\
\text { Strains }\end{array}$ & $\begin{array}{c}\text { Other } \\
\text { Sprains and } \\
\text { Strains }\end{array}$ & $\begin{array}{c}\text { Upper } \\
\text { Extremity } \\
\text { Neurologic }\end{array}$ & $\begin{array}{c}\text { Other } \\
\text { Injuries }\end{array}$ \\
\hline \multicolumn{9}{|l|}{ MME for all claims } \\
\hline \multirow[t]{2}{*}{ Must-access PDMP } & 2.1268 & 3.4728 & -46.3165 & $-160.4689 * *$ & $-33.6107 * *$ & $-13.3391^{*}$ & 3.1276 & -6.6082 \\
\hline & $(17.9836)$ & $(3.0668)$ & $(28.2368)$ & $(66.0131)$ & (14.6385) & (7.8328) & (35.0599) & $(7.9022)$ \\
\hline Observations & 1,118 & 1,118 & 1,118 & 1,118 & 1,118 & 1,118 & 1,118 & 1,118 \\
\hline State-specific trends & No & No & No & No & No & No & No & No \\
\hline Mean of outcome variable & 354.6 & 34.3 & 360.1 & 1232.3 & 162.6 & 139.1 & 242.1 & 123.9 \\
\hline$\%$ effect for PDMPs & $1 \%$ & $10 \%$ & $-13 \%$ & $-13 \% * *$ & $-21 \% * *$ & $-10 \% *$ & $1 \%$ & $-5 \%$ \\
\hline \multicolumn{9}{|c|}{ Number of non-opioid pain medication prescriptions } \\
\hline \multirow[t]{2}{*}{ Must-access PDMP } & 0.0319 & $0.0163^{* *}$ & $0.1173^{*}$ & $0.3401 * * *$ & 0.0870 & $0.0719 *$ & 0.2045 & $0.0413^{* *}$ \\
\hline & $(0.0282)$ & $(0.0076)$ & $(0.0603)$ & $(0.1173)$ & $(0.0525)$ & $(0.0367)$ & $(0.1304)$ & $(0.0183)$ \\
\hline Observations & 1,118 & 1,118 & 1,118 & 1,118 & 1,118 & 1,118 & 1,118 & 1,118 \\
\hline Mean of outcome variable & 0.533 & 0.2662 & 1.1958 & 2.4916 & 0.9211 & 0.702 & 0.9732 & 0.4039 \\
\hline$\%$ effect for PDMPs & $6 \%$ & $6 \% * *$ & $10 \% *$ & $14 \% * * *$ & $9 \%$ & $10 \% *$ & $21 \%$ & $10 \% * *$ \\
\hline \multicolumn{9}{|c|}{ Any active physical medicine services } \\
\hline \multirow[t]{2}{*}{ Must access PDMP } & 0.0151 & 0.0022 & 0.0114 & 0.0131 & 0.0057 & 0.0111 & 0.0201 & 0.0039 \\
\hline & $(0.0134)$ & $(0.0016)$ & $(0.0128)$ & $(0.0219)$ & $(0.0105)$ & $(0.0086)$ & $(0.0136)$ & $(0.0036)$ \\
\hline Observations & 1,118 & 1,118 & 1,118 & 1,118 & 1,118 & 1,118 & 1,118 & 1,118 \\
\hline State-specific trends & No & Yes & No & No & No & No & No & No \\
\hline Mean of outcome variable & 0.4148 & 0.0805 & 0.5688 & 0.7192 & 0.4259 & 0.3902 & 0.4866 & 0.1164 \\
\hline$\%$ effect for PDMPs & $4 \%$ & $3 \%$ & $2 \%$ & $2 \%$ & $1 \%$ & $3 \%$ & $4 \%$ & $3 \%$ \\
\hline \multicolumn{9}{|c|}{ Number of visits for active physical medicine services } \\
\hline \multirow[t]{2}{*}{ Must-access PDMP } & 0.2882 & 0.0237 & 0.2397 & -0.2028 & 0.0488 & 0.1646 & -0.0123 & 0.1075 \\
\hline & $(0.3407)$ & $(0.0228)$ & $(0.3232)$ & $(0.4847)$ & $(0.1173)$ & $(0.1614)$ & $(0.2415)$ & $(0.0769)$ \\
\hline Observations & 1,118 & 1,118 & 1,118 & 1,118 & 1,118 & 1,118 & 1,118 & 1,118 \\
\hline State-specific trends & No & No & No & No & No & No & No & No \\
\hline Mean of outcome variable & 7.4671 & 0.6944 & 10.4126 & 12.0179 & 3.9673 & 5.2183 & 6.2638 & 1.8177 \\
\hline \multirow[t]{2}{*}{ Must-access PDMP } & 0.0048 & $0.0018^{* * *}$ & 0.0103 & $0.0217^{*}$ & 0.0004 & 0.0023 & 0.0043 & 0.0024 \\
\hline & $(0.0036)$ & $(0.0005)$ & $(0.0075)$ & $(0.0115)$ & $(0.0016)$ & $(0.0028)$ & $(0.0133)$ & $(0.0017)$ \\
\hline Observations & 1,118 & 1,118 & 1,118 & 1,118 & 1,118 & 1,118 & 1,118 & 1,118 \\
\hline State-specific trends & No & No & No & No & No & No & No & No \\
\hline Mean of outcome variable & 0.1638 & 0.0178 & 0.4138 & 0.4616 & 0.0568 & 0.132 & 0.2783 & 0.0537 \\
\hline$\%$ effect for PDMPs & $3 \%$ & $10 \% * * *$ & $2 \%$ & $5 \% *$ & $1 \%$ & $2 \%$ & $2 \%$ & $4 \%$ \\
\hline \multicolumn{9}{|c|}{ Number of visits for interventional pain management services } \\
\hline \multirow[t]{2}{*}{ Must-access PDMP } & 0.0029 & $0.0022 * * *$ & 0.0221 & $0.0624^{*}$ & -0.0050 & 0.0030 & 0.0143 & 0.0024 \\
\hline & $(0.0060)$ & $(0.0007)$ & $(0.0178)$ & $(0.0352)$ & $(0.0056)$ & $(0.0047)$ & $(0.0245)$ & $(0.0039)$ \\
\hline Observations & 1,118 & 1,118 & 1,118 & 1,118 & 1,118 & 1,118 & 1,118 & 1,118 \\
\hline State-specific trends & No & No & No & No & No & No & No & No \\
\hline Mean of outcome variable & 0.2296 & 0.0233 & 0.6995 & 1.0663 & 0.1098 & 0.2003 & 0.4191 & 0.0923 \\
\hline$\%$ effect for PDMPs & $1 \%$ & $9 \% * * *$ & $3 \%$ & $6 \% *$ & $-5 \%$ & $1 \%$ & $3 \%$ & $3 \%$ \\
\hline
\end{tabular}

Notes: Estimates from state-level regressions for claims at 12 months of maturity, for injuries occurring October 2009-March 2018. Sample includes all claims aggregated to quarter of injury and state. Each observation is weighted by the number of claims represented. Controls are included for industry composition, average county-level unemployment rate, average county-level median household income, average countylevel percentage disabled, and average county-level percentage without health insurance. We also control for state and quarter fixed effects. ${ }^{*}$, $* *, * * *$ Statistically significant at the 10 percent, 5 percent, and 1 percent level, respectively. (The asterisks reported for the "\% effect" rows are the same as for the corresponding coefficient estimates.) Standard errors are clustered by state. To account for partial exposure for injuries for which the policy changed during the 12 months the data capture, we define the variable denoting the policy change as 1 if it was in effect for all 12 months of the postinjury exposure, 0.75 if it was in effect for 3 of the 4 quarters of postinjury exposure, 0.5 if it was in effect for 2 of the 4 quarters of postinjury exposure, and 0.25 if it was in effect for the last quarter of the 4 quarters of postinjury exposure.

Key: MME: morphine milligram equivalent amount of opioids; PDMP: must-access prescription drug monitoring program. 


\begin{tabular}{|c|c|c|c|}
\hline Outcome & $\begin{array}{c}\text { Weeks of Temporary } \\
\text { Disability Payments }\end{array}$ & $\begin{array}{c}\text { Weeks of Temporary } \\
\text { Disability Payments }\end{array}$ & $\begin{array}{c}\text { Claims with More Than } 7 \\
\text { Days of Lost Work Time }\end{array}$ \\
\hline \multicolumn{4}{|c|}{$\begin{array}{l}\text { Claims with More Than } \\
7 \text { Days of Lost Work }\end{array}$} \\
\hline Sample & Time & All Claims & All Claims \\
\hline \multicolumn{4}{|l|}{ A. Two policy specifications } \\
\hline \multirow[t]{2}{*}{ Must-access PDMP } & 0.0284 & 0.0053 & -0.0011 \\
\hline & $(0.1702)$ & $(0.0580)$ & $(0.0026)$ \\
\hline \multirow[t]{2}{*}{ Limits on initial opioid Rx } & $0.4422^{*}$ & $0.2366 * *$ & $0.0101 * *$ \\
\hline & $(0.2260)$ & $(0.0866)$ & $(0.0046)$ \\
\hline Observations & 1,118 & 1,118 & 1,118 \\
\hline Mean of outcome variable & 13.3 & 2.830 & 0.212 \\
\hline$\%$ effect for PDMPs & $0 \%$ & $0 \%$ & $-1 \%$ \\
\hline$\%$ effect for initial limits & $3 \% *$ & $8 \% * *$ & $5 \% * *$ \\
\hline \multicolumn{4}{|c|}{ B. Two policy specifications with state-specific time trends } \\
\hline \multirow[t]{2}{*}{ Must-access PDMP } & 0.0648 & 0.0254 & -0.0001 \\
\hline & $(0.1546)$ & $(0.0528)$ & $(0.0022)$ \\
\hline \multirow[t]{2}{*}{ Limits on initial opioid $R x$} & 0.2609 & 0.1085 & 0.0038 \\
\hline & $(0.2077)$ & $(0.0905)$ & $(0.0037)$ \\
\hline Observations & 1,118 & 1,118 & 1,118 \\
\hline Mean of outcome variable & 13.3 & 2.830 & 0.212 \\
\hline$\%$ effect for PDMPs & $0 \%$ & $1 \%$ & $0 \%$ \\
\hline$\%$ effect for initial limits & $2 \%$ & $4 \%$ & $2 \%$ \\
\hline \multicolumn{4}{|c|}{ C. Specifications for PDMPs only } \\
\hline \multirow[t]{2}{*}{ Must-access PDMP } & 0.1455 & 0.0622 & 0.0013 \\
\hline & $(0.1605)$ & $(0.0638)$ & $(0.0029)$ \\
\hline Observations & 1,118 & 1,118 & 1,118 \\
\hline Mean of outcome variable & 13.3 & 2.830 & 0.212 \\
\hline$\%$ effect for PDMPs & $1 \%$ & $2 \%$ & $1 \%$ \\
\hline
\end{tabular}

Notes: Estimates from state-level regressions for claims at 12 months of maturity, for injuries occurring October 2009March 2018. Samples include all claims or claims with more than 7 days of lost work time (as indicated) aggregated to quarter of injury and state. Each observation is weighted by the number of claims represented. Controls are included for industry composition, average county-level unemployment rate, average county-level median household income, average county-level percentage disabled, and average county-level percentage without health insurance. We also control for state and quarter fixed effects. $*, * *, * * *$ Statistically significant at the 10 percent, 5 percent, and 1 percent level, respectively. (The asterisks reported for the "\% effect" rows are the same as for the corresponding coefficient estimates.) Standard errors are clustered by state. To account for partial exposure for injuries for which the policy changed during the 12 months the data capture, we define the variable denoting the policy change as 1 if it was in effect for all 12 months of the postinjury exposure, 0.75 if it was in effect for 3 of the 4 quarters of postinjury exposure, 0.5 if it was in effect for 2 of the 4 quarters of postinjury exposure, and 0.25 if it was in effect for only the last quarter of the 4 quarters of postinjury exposure.

Key: PDMP: must-access prescription drug monitoring program; Rx: prescription. 